\title{
Quarterly Report of RCRA Groundwater Monitoring Data for Period April 1, 1991 through June 30, 1991
}

\section{Geosciences Group}

Westinghouse Hanford Company

Environmental Division

Date Publis'i ifad

September 1991

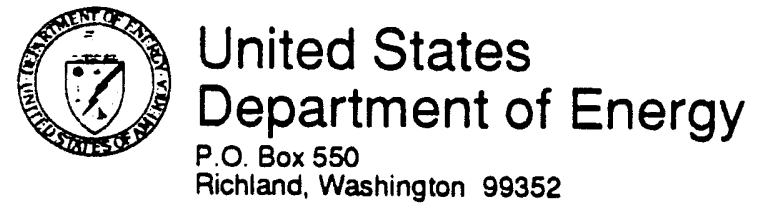

Richland, Washington 99352 
$\mathrm{DOE} / \mathrm{RL}-91-47$

\section{CONTENTS}

1.0 INTRODUCTION . . . . . . . . . . . . . . . . . . . . . . . . 1

2.0300 AREA PROCESS TRENCHES . . . . . . . . . . . . . . . . . 5

$3.0183-H$ SOLAR EVAPORATION BASINS . . . . . . . . . . . . . . 11

4.0200 AREAS LOW-LEVEL BURIAL GROUNDS . . . . . . . . . . . . . . 17

$5.0100-N$ AREA RCRA SITES . . . . . . . . . . . . . . . . . . . . 23

$6.0216-\mathrm{A}-29 \mathrm{DITCH}$. . . . . . . . . . . . . . . . . . . . 29

7.0 216-A-10 Crib...................... . . . 33

8.0 216-A-36B Crib ..................... 35

9.0 216-B-3 POND SYSTEM . . . . . . . . . . . . . . . . . . . 37

10.0 2101-M POND . . . . . . . . . . . . . . . . . . . . . . 41

11.0 GROUT TREATMENT FACILITY . . . . . . . . . . . . . . . . . . . . 43

12.0 SINGLE-SHELL TANKS . . . . . . . . . . . . . . . . . . 47

13.0 NONRADIOACTIVE DANGEROUS WASTE LANDFILL . . . . . . . . . . . . 51

14.0 REFERENCES . . . . . . . . . . . . . . . . . . . . . 55 
DOE/RL-91-47

\section{LIST OF FIGURES}

1-1 Locations of the RCRA Groundwater Monitoring Projects and Landmarks on the Hanford Site ................. 3

\section{LIST OF TABLES}

2-1 RCRA Water Level Measurement Report 300 Area Process Trenches Second Quarter 1991...................... 6

3-1 RCRA Water Level Measurement Report 183-H Solar Evaporation Basins - Second Quarter 1991 . . . . . . . . . . . . . . 12

4-1 RCRA Water Level Measurement Report Low-Level Burial Grounds Second Quarter 1991................... 18

5-1 RCRA Water Level Measurement Report 100-N Area - Second Quarter 1991 . . . . . . . . . . . . . . . . . . 24

6-1 RCRA Water Level Measurement Report 216-A-29 Ditch - Second Quarter 1991 . . . . . . . . . . . . . . . . 30

7-1 RCRA Water Level Measurement Report 216-A-10 Crib - Second Quarter 1991 ....................... . 34

8-1 RCRA Water Level Measurement Report 216-A-36B Crib - Second Quarter 1991 ....................... 36

9-1 RCRA Water Level Measurement Report 216-B-3 Pond System Second Quarter 1991 ................... . 38

10-1 RCRA Water Level Measurement Report 2101-M Pond - Second Quarter 1991 ....................... 42

11-1 RCRA Water Level Measurement Report Grout Treatment Facility Second Quarter 1991 . . . . . . . . . . . . . . . . . 44

12-1 RCRA Water Level Measurement Report Single-Shell Tanks Second Quarter 1991 .................... . . 48

13-1 RCRA Water Level Measurement Report NRDWL - Second Quarter 1991 . 52 
$\mathrm{DOE} / \mathrm{RL}-91-47$

\section{ACRONYMS}

CERCLA Comprehensive Environmental Response, Compensation and Liability

CLP

HEHF

LLWMA

Contract Laboratory Program

NRDWL

Hanford Environmental Health Foundation

Low-Level Waste Management Area

PNL

QA

Nonradioactive Dangerous Waste Landfill

Pacific Northwest Laboratory

QC

quality assurance

RCRA

SST

UST

WMA

Resource Conservation and Recovery Act of 1976

Single-Shell Tanks

United States Testing Company, Inc.

Waste Management Area 
DOE/RL-91-47

This page intentionally left blank. 


\subsection{INTRODUCTION}

Hanford Site interim-status groundwater monitoring projects are conducted as either background, indicator parameter evaluation, or groundwater quality assessment monitoring programs as defined in the Resource Conservation and Recovery Act of 1976 (RCRA); and 40 CFR 265, Interim Status Standards for Owners and Operators of Hazardous Waste Treatment, Storage, and Disposal Facilities, as amended (EPA 1989). Compliance with the 40 CFR 265 regulations are required by the Washington Administrative Code 173-303 (Ecology 1991). This submittal provides data obtained from groundwater monitoring activities for April 1, 1991 through June 30, 1991.

On June 1, 1990, Pacific Northwest Laboratory (PNL) announced the termination of its subcontract with United States Testing Company, Inc. (UST), which was responsible for analyzing chemistry samples and providing the resulting data. The U.S. Department of Energy Field Office, Richland, currently is in the process of awarding a contract to a suitable analytical laboratory.

In February 1991, a list of wells was assembled for priority sampling. The 1 ist includes 52 groundwater monitoring wells from RCRA and operational groundwater monitoring programs across the Hanford Site. This list, along with the constituents analyzed for, were presented in the previous quarterly report (DOE-RL 1991b).

The samples were collected in March and April 1991 and submitted to laboratories contracted to perform Comprehensive Environmental Response, Compensation, and Liability Act of 1980 (CERCLA) analyses. Laboratory analyses performed under the Contract Laboratory Program (CLP), the program specified for CERCLA, vary from the requirements for RCRA analyses. Although the CLP data validation requirements exceed those of RCRA, the CLP contract does not include all of the RCRA constituents.

These water sampling data were collected to assess any hydrochemical changes that may have occurred, because sampling ceased last year. The data are being used for information purposes only to aid in groundwater flow and contaminant transport interpretation. The data are not included in this quarterly report because they were not produced in accordance with RCRA protocol. This information is available on request.

Groundwater sampling was resumed on the Hanford Site on June 6, 1991. The laboratory that is performing the majority of the analyses, under an interim contract, is IT Analytical Services. Nitrates analys is is being performed by PNL, and the Hanford Environmental and Health Foundation (HEHF) will be performing coloform analys is in the future. Currently, no laboratory is available to perform the inductively coupled plasma method for metals detection. These samples are being archived for future analysis. Westinghouse Hanford Company is in the process of initiating an internal quality assurance/quality control ( $Q A / Q C$ ) laboratory to evaluate the PNL primary contract laboratory performance. 
Three RCRA facilities had groundwater samples collected during the month of June. A complete set of data has not be received for these facilities; the data will be reported in the next quarterly report (for period July 1991 through September 1991).

Water level data have continued to be collected at each of the facilities. This report contains the water level measurements from 14 Hanford Site RCRA groundwater projects. The location of each facility is shown in Figure 1-1. Water level measurements have been collected for the Liquid Effluent Retention Facility, 216-S-10 Facility, 216-U-12 Crib, 216-B-63 Trench, and 100-D Area. These facilities have not initiated the background monitoring program; therefore, the measurements are not provided in this report.

The water levels were collected in accordance with the Hanford Site approved procedure found in WHC-CM-7-7 (WHC 1991). Water level measurements were obtained using standardized, weighted, steel measuring tapes. Readings were repeated until a consistent measurement was obtained. Readings are considered consistent if they are within $+/-0.02 \mathrm{ft}$.

The measuring device was decontaminated before each series of measurements and after each well measurement to ensure the safety of personnel and to prevent cross-contamination of wells.

The number of significant digits listed in the water level tables is not indicative of the accuracy.

Most facilities have not had any changes to their monitoring networks. Well location maps for these facilities can be found in the quarterly report for the period July 1 through September 30, 1990 (DOE-RL 1990). Any changes made to monitoring networks for this period are identified in the following sections. 
DOE/RL-91-47

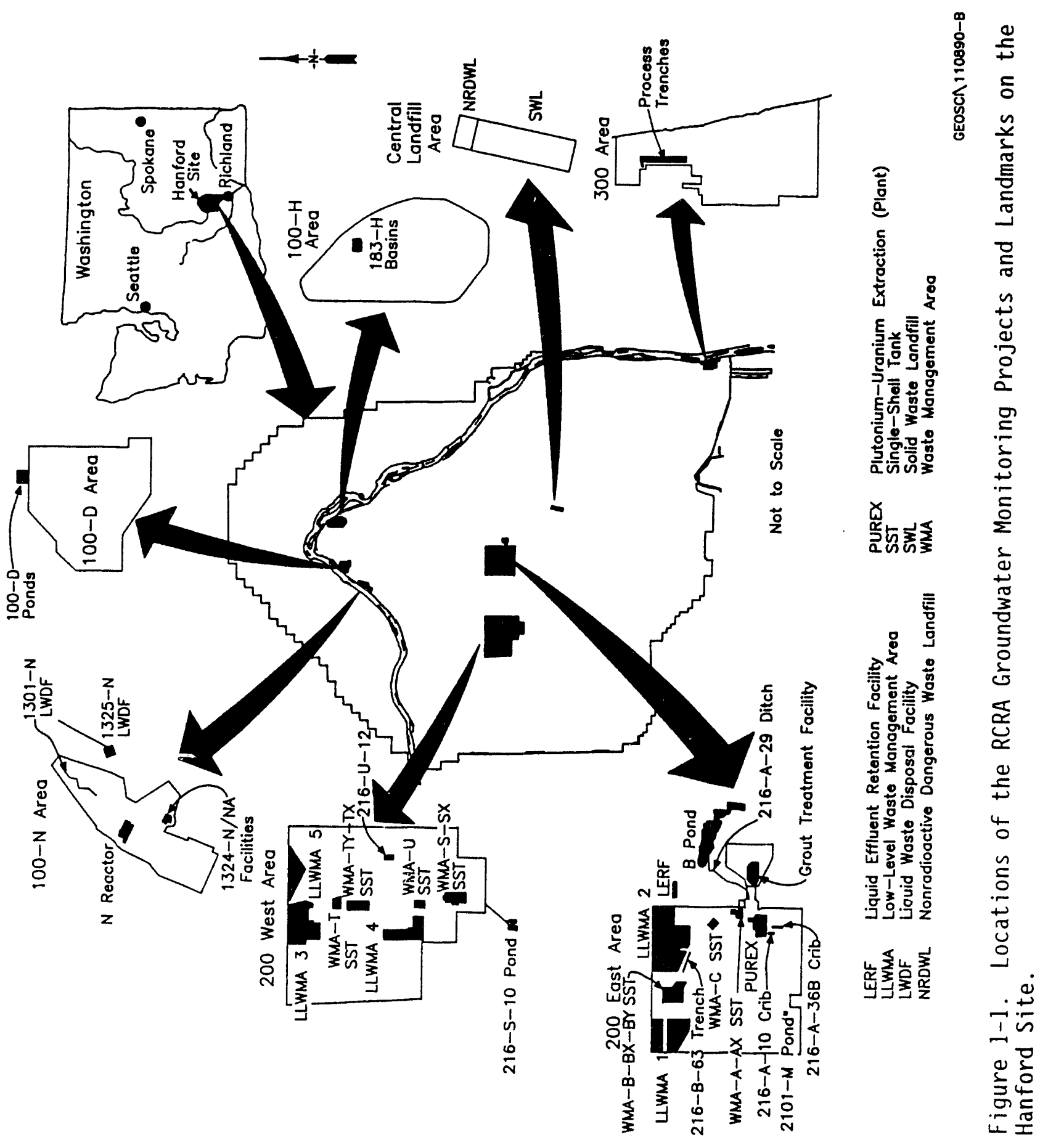


DOE/RL-91-47

This page intentionally left blank. 
$\mathrm{DOE} / \mathrm{RL}-91-47$

\subsection{AREA PROCESS TRENCHES}

\section{J. V. Borghese}

\section{Pacific Northwest Laboratory}

The groundwater monitoring network for this RCRA facility has not changed from that described in the quarterly report for the period July 1 through

September 30, 1990 (DOE-RL 1990). Please refer to that repoit for a description of the facility and the monitoring network.

Water level data for wells in the vicinity of the trenches for the second quarter of 1991 are presented in Table 2-1. 
Table 2-1. RCRA water Level Measurement Report 300 Area

Process Trenches - Second Quarter 1991. (sheet 1 of 4)

\begin{tabular}{|c|c|c|c|}
\hline We11 & Date & $\begin{array}{c}\text { Depth to water } \\
(\mathrm{ft})\end{array}$ & $\begin{array}{c}\text { Water level el evation } \\
\text { above } \mathrm{ms} \text { i (ft) }\end{array}$ \\
\hline \multicolumn{4}{|c|}{ Wells Monitoring the Top of the Unconfined Aquifer } \\
\hline $399-1-1$ & $\begin{array}{l}4 / 25 / 91 \\
5 / 29 / 91 \\
6 / 18 / 91\end{array}$ & $\begin{array}{l}29.44 \\
28.82 \\
28.25\end{array}$ & $\begin{array}{l}346.46 \\
347.08 \\
347.65\end{array}$ \\
\hline $399-1-10$ & $\begin{array}{l}4 / 01 / 91 \\
4 / 25 / 91 \\
5 / 29 / 91 \\
6 / 18 / 91\end{array}$ & $\begin{array}{l}28.65 \\
27.04 \\
26.09 \\
26.86\end{array}$ & $\begin{array}{l}345.00 \\
346.61 \\
347.56 \\
346.79\end{array}$ \\
\hline $399-1-11$ & $\begin{array}{l}4 / 25 / 91 \\
5 / 29 / 91 \\
6 / 18 / 91\end{array}$ & $\begin{array}{l}31.30 \\
30.38 \\
31.00\end{array}$ & $\begin{array}{l}346.49 \\
347.41 \\
346.79\end{array}$ \\
\hline $399-1-12$ & $\begin{array}{l}4 / 25 / 91 \\
5 / 29 / 91 \\
6 / 18 / 91\end{array}$ & $\begin{array}{l}38.39 \\
37.47 \\
37.90\end{array}$ & $\begin{array}{l}346.06 \\
346.98 \\
346.55\end{array}$ \\
\hline $399-1-13$ & $\begin{array}{l}4 / 01 / 91 \\
4 / 25 / 91 \\
5 / 29 / 91 \\
6 / 18 / 91\end{array}$ & $\begin{array}{l}43.76 \\
42.58 \\
41.66 \\
41.98\end{array}$ & $\begin{array}{l}344.86 \\
346.04 \\
346.96 \\
346.64\end{array}$ \\
\hline $399-1-14$ & $\begin{array}{l}4 / 25 / 91 \\
5 / 29 / 91 \\
6 / 18 / 91\end{array}$ & $\begin{array}{l}36.93 \\
36.04 \\
36.25\end{array}$ & $\begin{array}{l}346.30 \\
347.19 \\
346.98\end{array}$ \\
\hline $399-1-15$ & $\begin{array}{l}4 / 25 / 91 \\
5 / 29 / 91 \\
6 / 18 / 91\end{array}$ & $\begin{array}{l}33.08 \\
32.27 \\
32.49\end{array}$ & $\begin{array}{l}346.51 \\
347.32 \\
347.10\end{array}$ \\
\hline $399-1-16 A$ & $\begin{array}{l}4 / 01 / 91 \\
4 / 25 / 91 \\
5 / 29 / 91 \\
6 / 18 / 91\end{array}$ & $\begin{array}{l}37.13 \\
35.54 \\
34.68 \\
35.32\end{array}$ & $\begin{array}{l}344.38 \\
345.97 \\
346.83 \\
346.19\end{array}$ \\
\hline $399-1-17 A$ & $\begin{array}{l}4 / 01 / 91 \\
4 / 25 / 91 \\
5 / 29 / 91 \\
6 / 18 / 91 \\
6 / 24 / 91\end{array}$ & $\begin{array}{l}32.94 \\
31.56 \\
30.67 \\
31.15 \\
33.39\end{array}$ & $\begin{array}{l}344.53 \\
345.91 \\
346.80 \\
346.32 \\
344.18 \star\end{array}$ \\
\hline $399-1-18 A$ & $\begin{array}{l}4 / 01 / 91 \\
4 / 25 / 91 \\
5 / 29 / 91 \\
6 / 18 / 91\end{array}$ & $\begin{array}{l}45.11 \\
44.10 \\
43.33 \\
43.29\end{array}$ & $\begin{array}{l}345.72 \\
346.73 \\
347.50 \\
347.54\end{array}$ \\
\hline
\end{tabular}


Table 2-1. RCRA Water Level Measurement Report 300 Area

Process Trenches - Second Quarter 1991. (sheet 2 of 4)

\begin{tabular}{|c|c|c|c|}
\hline Well & Date & $\begin{array}{c}\text { Depth to water } \\
(\mathrm{ft})\end{array}$ & $\begin{array}{c}\text { Water level elevation } \\
\text { above msl (ft) }\end{array}$ \\
\hline $399-1-19$ & $\begin{array}{l}4 / 25 / 91 \\
5 / 29 / 91 \\
6 / 18 / 91\end{array}$ & $\begin{array}{l}28.62 \\
27.70 \\
28.36\end{array}$ & $\begin{array}{l}346.02 \\
346.94 \\
346.28\end{array}$ \\
\hline $399-1-3$ & $\begin{array}{l}4 / 25 / 91 \\
5 / 29 / 91 \\
6 / 18 / 91\end{array}$ & $\begin{array}{l}38.76 \\
37.86 \\
38.50\end{array}$ & $\begin{array}{l}345.97 \\
346.87 \\
346.23\end{array}$ \\
\hline $399-1-4$ & $\begin{array}{l}4 / 25 / 91 \\
5 / 29 / 91 \\
6 / 18 / 91\end{array}$ & $\begin{array}{l}34.21 \\
33.28 \\
33.64\end{array}$ & $\begin{array}{l}346.90 \\
347.83 \\
347.47\end{array}$ \\
\hline $399-1-5$ & $\begin{array}{l}4 / 25 / 91 \\
5 / 29 / 91 \\
6 / 18 / 91\end{array}$ & $\begin{array}{l}33.68 \\
32.75 \\
33.44\end{array}$ & $\begin{array}{l}346.14 \\
347.07 \\
346.38\end{array}$ \\
\hline $399-1-7$ & $\begin{array}{l}4 / 25 / 91 \\
5 / 29 / 91 \\
6 / 18 / 91\end{array}$ & $\begin{array}{l}39.59 \\
38.82 \\
39.44\end{array}$ & $\begin{array}{l}345.94 \\
346.81 \\
346.19\end{array}$ \\
\hline $399-1-8$ & $\begin{array}{l}4 / 25 / 91 \\
5 / 29 / 91 \\
6 / 18 / 91\end{array}$ & $\begin{array}{l}38.99 \\
38.10 \\
38.69\end{array}$ & $\begin{array}{l}345.92 \\
346.81 \\
346.22\end{array}$ \\
\hline $399-2-1$ & $\begin{array}{l}4 / 25 / 91 \\
5 / 29 / 91 \\
6 / 18 / 91\end{array}$ & $\begin{array}{l}29.28 \\
28.36 \\
29.13\end{array}$ & $\begin{array}{l}345.98 \\
346.90 \\
346.13\end{array}$ \\
\hline $399-2-2$ & $\begin{array}{l}4 / 25 / 91 \\
5 / 29 / 91 \\
6 / 18 / 91\end{array}$ & $\begin{array}{l}29.55 \\
28.69 \\
29.34\end{array}$ & $\begin{array}{l}345.98 \\
346.84 \\
346.19\end{array}$ \\
\hline $399-2-3$ & $\begin{array}{l}4 / 25 / 91 \\
5 / 29 / 91 \\
6 / 18 / 91\end{array}$ & $\begin{array}{l}29.49 \\
28.62 \\
29.30\end{array}$ & $\begin{array}{l}345.95 \\
346.82 \\
346.14\end{array}$ \\
\hline $399-3-1$ & $\begin{array}{l}4 / 25 / 91 \\
5 / 29 / 91 \\
6 / 18 / 91\end{array}$ & $\begin{array}{l}38.55 \\
37.65 \\
38.46\end{array}$ & $\begin{array}{l}345.88 \\
346.78 \\
345.97\end{array}$ \\
\hline $399-3-10$ & $\begin{array}{l}4 / 25 / 91 \\
5 / 29 / 91 \\
6 / 18 / 91\end{array}$ & $\begin{array}{l}39.60 \\
38.73 \\
39.59\end{array}$ & $\begin{array}{l}345.80 \\
346.67 \\
345.81\end{array}$ \\
\hline $399-3-12$ & $\begin{array}{l}4 / 25 / 91 \\
5 / 29 / 91 \\
6 / 18 / 91\end{array}$ & $\begin{array}{l}40.59 \\
39.73 \\
40.38\end{array}$ & $\begin{array}{l}345.79 \\
346.65 \\
346.00\end{array}$ \\
\hline
\end{tabular}


Table 2-1. RCRA Water Level Measurement Report 300 Area Process Trenches - Second Quarter 1991. (sheet 3 of 4)

\begin{tabular}{|c|c|c|c|}
\hline Well & Date & $\begin{array}{c}\text { Depth to water } \\
(\mathrm{ft})\end{array}$ & $\begin{array}{c}\text { Water level elevation } \\
\text { above msl (ft) }\end{array}$ \\
\hline $399-3-6$ & $\begin{array}{l}4 / 25 / 91 \\
5 / 29 / 91 \\
6 / 18 / 91\end{array}$ & $\begin{array}{l}47.07 \\
46.25 \\
46.63\end{array}$ & $\begin{array}{l}345.78^{\prime} \\
346.60 \\
346.22\end{array}$ \\
\hline $399-3-7$ & $\begin{array}{l}4 / 25 / 91 \\
5 / 29 / 91 \\
\epsilon / 18 / 91\end{array}$ & $\begin{array}{l}48.41 \\
47.57 \\
48.08\end{array}$ & $\begin{array}{l}345.81 \\
346.65 \\
346.14\end{array}$ \\
\hline $399-3-9$ & $\begin{array}{l}4 / 25 / 91 \\
5 / 29 / 91 \\
6 / 18 / 91\end{array}$ & $\begin{array}{l}41.45 \\
47.59 \\
41.39\end{array}$ & $\begin{array}{l}345.86 \\
346.72 \\
345.92\end{array}$ \\
\hline $399-4-1$ & $\begin{array}{l}4 / 25 / 91 \\
5 / 29 / 91 \\
6 / 18 / 91\end{array}$ & $\begin{array}{l}49.30 \\
48.46 \\
49.29\end{array}$ & $\begin{array}{l}345.70 \\
346.54 \\
345.71\end{array}$ \\
\hline $399-4-10$ & $\begin{array}{l}4 / 25 / 91 \\
5 / 29 / 91 \\
6 / 18 / 91\end{array}$ & $\begin{array}{l}31.80 \\
30.94 \\
31.86\end{array}$ & $\begin{array}{l}345.79 \\
346.65 \\
345.73\end{array}$ \\
\hline $399-4-11$ & $\begin{array}{l}4 / 25 / 91 \\
5 / 29 / 91 \\
6 / 18 / 91\end{array}$ & $\begin{array}{l}58.80 \\
57.96 \\
58.58\end{array}$ & $\begin{array}{l}345.73 \\
346.57 \\
345.95\end{array}$ \\
\hline $399-4-7$ & $\begin{array}{l}4 / 25 / 91 \\
5 / 29 / 91 \\
6 / 18 / 91\end{array}$ & $\begin{array}{l}31.24 \\
30.40 \\
31.36\end{array}$ & $\begin{array}{l}345.75 \\
346.59 \\
345.63\end{array}$ \\
\hline $399-4-9$ & $\begin{array}{l}4 / 25 / 91 \\
5 / 29 / 91 \\
6 / 18 / 91\end{array}$ & $\begin{array}{l}35.57 \\
34.70 \\
35.58\end{array}$ & $\begin{array}{l}345.83 \\
346.70 \\
345.82\end{array}$ \\
\hline $399-5-1$ & $\begin{array}{l}4 / 25 / 91 \\
5 / 29 / 91 \\
6 / 18 / 91\end{array}$ & $\begin{array}{l}49.94 \\
49.15 \\
49.44\end{array}$ & $\begin{array}{l}345.66 \\
346.45 \\
346.16\end{array}$ \\
\hline $399-6-1$ & $\begin{array}{l}4 / 25 / 91 \\
5 / 29 / 91 \\
6 / 18 / 91\end{array}$ & $\begin{array}{l}41.18 \\
40.44 \\
40.37\end{array}$ & $\begin{array}{l}345.75 \\
346.49 \\
346.56\end{array}$ \\
\hline $399-8-1$ & $\begin{array}{l}4 / 25 / 91 \\
5 / 29 / 91 \\
6 / 18 / 91\end{array}$ & $\begin{array}{l}48.95 \\
48.20 \\
48.24\end{array}$ & $\begin{array}{l}345.93 \\
346.68 \\
346.64\end{array}$ \\
\hline $399-8-2$ & $\begin{array}{l}4 / 25 / 91 \\
5 / 29 / 91 \\
6 / 18 / 91\end{array}$ & $\begin{array}{l}50.18 \\
49.47 \\
49.01\end{array}$ & $\begin{array}{l}345.88 \\
346.59 \\
347.05\end{array}$ \\
\hline
\end{tabular}


Table 2-1. RCRA Water Level Measurement Report 300 Area Process Trenches - Second Quarter 1991. (sheet 4 of 4)

\begin{tabular}{cccc}
\hline Wel1 & Date & $\begin{array}{c}\text { Depth to water } \\
(\mathrm{ft})\end{array}$ & $\begin{array}{c}\text { Water level elevation } \\
\text { above ms } 1 \text { (ft) }\end{array}$ \\
\hline $399-8-3$ & $4 / 25 / 91$ & 47.01 & 346.10 \\
& $5 / 29 / 91$ & 46.25 & 346.86 \\
& $6 / 10 / .2$ & 46.17 & 346.94 \\
$699-527-E 14$ & $4 / 25 / 91$ & 54.10 & 345.66 \\
& $5 / 27 / 91$ & 53.28 & 346.48 \\
& $6 / 18 / 91$ & 54.11 & 345.65
\end{tabular}

Wells Monitoring the Bottom of the Unconfined Aquifer

\begin{tabular}{|c|c|c|c|}
\hline $399-1-16 B$ & $\begin{array}{l}4 / 25 / 91 \\
5 / 29 / 91 \\
6 / 18 / 91\end{array}$ & $\begin{array}{l}34.93 \\
34.10 \\
34.74\end{array}$ & $\begin{array}{l}346.21 \\
347.04 \\
346.40\end{array}$ \\
\hline $399-1-17 B$ & $\begin{array}{l}4 / 25 / 91 \\
5 / 29 / 91 \\
6 / 18 / 91\end{array}$ & $\begin{array}{l}31.80 \\
30.86 \\
31.39\end{array}$ & $\begin{array}{l}346.07 \\
347.01 \\
346.48\end{array}$ \\
\hline $399-1-18 B$ & $\begin{array}{l}4 / 25 / 91 \\
5 / 29 / 91 \\
6 / 18 / 91\end{array}$ & $\begin{array}{l}42.97 \\
42.20 \\
42.29\end{array}$ & $\begin{array}{l}346.97 \\
347.74 \\
347.65\end{array}$ \\
\hline \multicolumn{4}{|c|}{ Wells Monitoring the Confined Aquifer } \\
\hline $399-1-17 C$ & $\begin{array}{l}4 / 25 / 91 \\
5 / 29 / 91 \\
6 / 18 / 91\end{array}$ & $\begin{array}{l}2.00 \\
2.08 \\
2.32\end{array}$ & $\begin{array}{l}376.13 \\
376.05 \\
375.81\end{array}$ \\
\hline $399-1-18 C$ & $\begin{array}{l}4 / 25 / 91 \\
5 / 29 / 91 \\
6 / 18 / 91\end{array}$ & $\begin{array}{l}40.68 \\
39.93 \\
39.92\end{array}$ & $\begin{array}{l}347.37 \\
348.12 \\
348.13\end{array}$ \\
\hline $399-1-9$ & $\begin{array}{l}4 / 25 / 91 \\
5 / 29 / 91 \\
6 / 18 / 91\end{array}$ & $\begin{array}{l}19.24 \\
19.02 \\
19.41\end{array}$ & $\begin{array}{l}365.50 \\
365.78 \\
365.39\end{array}$ \\
\hline NOTES: 1. & \multicolumn{3}{|c|}{$\begin{array}{l}\text { Water level elevations are calculated by subtracting the } \\
\text { measured depth to water from the surveyed elevation for the } \\
\text { well. } \\
\text { Depth-to-water values are transcribed from field records. } \\
\text { Elevations marked with an } * *^{\prime} \text { were measured at the time of } \\
\text { sampling. }\end{array}$} \\
\hline
\end{tabular}


DOE/RL-91-47

This page intentionally left blank. 
DOE/RL-91-47

\subsection{3-H SOLAR EVAPORATION BASINS}

R. E. Peterson

Westinghouse Hanford Company

The groundwater monitoring network for this RCRA facility has not changed from that described in the quarterly report for the period July l through September 30, 1990 (DOE-RL 1990). Please refer to that report for a description of the facility and the monitoring network. Groundwater samples were collected in June. Results of the arialyses will be reported next quarter.

Water level data for wells in the vicinity of the basins for the second quarter of 1991 are presented in Table 3-1. 
Table 3-1. RCRA Water Level Measurement Report 183-H Solar Evaporation Basins - Second Quarter 1991. (sheet 1 of 5)

\begin{tabular}{|c|c|c|c|}
\hline Well & Date & $\begin{array}{l}\text { Depth to water } \\
(\mathrm{ft})\end{array}$ & $\begin{array}{l}\text { Water level elevation } \\
\text { above msl (ft) }\end{array}$ \\
\hline \multicolumn{4}{|c|}{$\begin{array}{l}\text { Wells Monitoring the Top of the Unconfined Aquifer } \\
\text { (Hanford Formation) }\end{array}$} \\
\hline $199-\mathrm{H} 3-1$ & $\begin{array}{l}4 / 11 / 91 \\
4 / 30 / 91 \\
5 / 16 / 91 \\
5 / 29 / 91 \\
6 / 06 / 91 \\
6 / 20 / 91\end{array}$ & $\begin{array}{l}43.77 \\
43.55 \\
43.45 \\
43.29 \\
44.08 \\
42.71\end{array}$ & $\begin{array}{l}377.71 \\
377.93 \\
378.03 \\
378.19 \\
377.40 * \\
378.77\end{array}$ \\
\hline $199-H 3-2 A$ & $\begin{array}{l}4 / 11 / 91 \\
4 / 11 / 91 \\
4 / 30 / 91 \\
5 / 16 / 91 \\
5 / 29 / 91 \\
6 / 06 / 91 \\
6 / 20 / 91\end{array}$ & $\begin{array}{l}40.09 \\
40.07 \\
39.78 \\
39.83 \\
39.42 \\
39.06 \\
38.75\end{array}$ & $\begin{array}{l}377.74^{\star} \\
377.75 \\
378.05 \\
378.00 \\
378.41 \\
378.77^{\star} \\
379.08\end{array}$ \\
\hline $199-H 3-2 B$ & $\begin{array}{l}4 / 11 / 91 \\
4 / 30 / 91 \\
5 / 16 / 91 \\
5 / 29 / 91 \\
6 / 20 / 91\end{array}$ & $\begin{array}{l}40.66 \\
40.34 \\
40.41 \\
39.99 \\
39.33\end{array}$ & $\begin{array}{l}377.76 \\
378.08 \\
378.01 \\
378.43 \\
379.09\end{array}$ \\
\hline $199-\mathrm{H} 4-10$ & $\begin{array}{l}4 / 11 / 91 \\
4 / 30 / 91 \\
5 / 16 / 91 \\
5 / 29 / 91 \\
6 / 20 / 91\end{array}$ & $\begin{array}{l}26.14 \\
26.58 \\
27.21 \\
24.52 \\
27.29\end{array}$ & $\begin{array}{l}378.30 \\
377.86 \\
377.23 \\
379.92 \\
377.15\end{array}$ \\
\hline $199-\mathrm{H} 4-11$ & $\begin{array}{l}4 / 11 / 91 \\
4 / 30 / 91 \\
5 / 16 / 91 \\
5 / 29 / 91 \\
6 / 07 / 91 \\
6 / 20 / 91\end{array}$ & $\begin{array}{l}38.88 \\
39.33 \\
39.03 \\
37.08 \\
36.72 \\
40.04\end{array}$ & $\begin{array}{l}377.96 \\
377.51 \\
377.81 \\
379.76 \\
380.12 * \\
376.80\end{array}$ \\
\hline $199-\mathrm{H} 4-12 \mathrm{~A}$ & $\begin{array}{l}4 / 11 / 91 \\
4 / 11 / 91 \\
4 / 30 / 91 \\
5 / 16 / 91 \\
5 / 29 / 91 \\
6 / 07 / 91 \\
6 / 20 / 91\end{array}$ & $\begin{array}{l}35.59 \\
35.32 \\
35.82 \\
35.23 \\
33.38 \\
33.23 \\
36.73\end{array}$ & $\begin{array}{l}377.91^{\star} \\
378.18 \\
377.68 \\
378.27 \\
380.12 \\
380.27 \star \\
376.77\end{array}$ \\
\hline
\end{tabular}


$\mathrm{DOE} / \mathrm{RL}-91-47$

Table 3-1. RCRA Water Level Measurement Report 183-H Solar Evaporation Basins - Second Quarter 1991. (sheet 2 of 5)

\begin{tabular}{|c|c|c|c|}
\hline Well & Date & $\begin{array}{c}\text { Depth to water } \\
(\mathrm{ft})\end{array}$ & $\begin{array}{c}\text { Water level elevation } \\
\text { above msl (ft) }\end{array}$ \\
\hline $199-\mathrm{H} 4-12 \mathrm{~B}$ & $\begin{array}{l}4 / 11 / 91 \\
4 / 30 / 91 \\
5 / 16 / 91 \\
5 / 29 / 91 \\
6 / 20 / 91\end{array}$ & $\begin{array}{l}35.41 \\
35.83 \\
35.35 \\
33.46 \\
36.61\end{array}$ & $\begin{array}{l}378.11 \\
377.69 \\
378.17 \\
380.06 \\
376.91\end{array}$ \\
\hline $199-\mathrm{H} 4-13$ & $\begin{array}{l}4 / 11 / 91 \\
4 / 20 / 91 \\
5 / 16 / 91 \\
5 / 29 / 91 \\
6 / 20 / 91\end{array}$ & $\begin{array}{l}40.26 \\
40.93 \\
40.47 \\
38.69 \\
41.73\end{array}$ & $\begin{array}{l}377.94 \\
377.27 \\
377.73 \\
379.51 \\
376.47\end{array}$ \\
\hline $199-\mathrm{H} 4-14$ & $\begin{array}{l}4 / 11 / 91 \\
4 / 30 / 91 \\
5 / 16 / 91 \\
5 / 29 / 91 \\
6 / 07 / 91 \\
6 / 20 / 91\end{array}$ & $\begin{array}{l}43.02 \\
42.68 \\
42.80 \\
42.27 \\
41.87 \\
41.64\end{array}$ & $\begin{array}{l}377.57 \\
377.91 \\
377.79 \\
378.32 \\
378.72 * \\
378.95\end{array}$ \\
\hline $199-H 4-15 A$ & $\begin{array}{l}4 / 11 / 91 \\
4 / 30 / 91 \\
5 / 16 / 91 \\
5 / 29 / 91 \\
6 / 20 / 91\end{array}$ & $\begin{array}{l}29.03 \\
29.28 \\
29.02 \\
27.02 \\
30.02\end{array}$ & $\begin{array}{l}378.18 \\
377.93 \\
378.19 \\
380.19 \\
377.19\end{array}$ \\
\hline $199-\mathrm{H} 4-15 B$ & $\begin{array}{l}4 / 11 / 91 \\
4 / 30 / 91 \\
5 / 16 / 91 \\
5 / 29 / 91 \\
6 / 20 / 91\end{array}$ & $\begin{array}{l}28.73 \\
29.02 \\
28.80 \\
26.86 \\
29.74\end{array}$ & $\begin{array}{l}378.19 \\
377.90 \\
378.12 \\
380.06 \\
377.18\end{array}$ \\
\hline $199-\mathrm{H} 4-16$ & $\begin{array}{l}4 / 11 / 91 \\
4 / 30 / 91 \\
5 / 16 / 91 \\
5 / 29 / 91 \\
6 / 07 / 91 \\
6 / 20 / 91\end{array}$ & $\begin{array}{l}47.11 \\
46.76 \\
46.89 \\
46.30 \\
45.94 \\
45.73\end{array}$ & $\begin{array}{l}377.12 \\
377.47 \\
377.34 \\
377.93 \\
378.29 \star \\
378.50\end{array}$ \\
\hline $199-\mathrm{H} 4-17$ & $\begin{array}{l}4 / 11 / 91 \\
4 / 30 / 91 \\
5 / 16 / 91 \\
5 / 29 / 91 \\
6 / 20 / 91\end{array}$ & $\begin{array}{l}41.56 \\
41.08 \\
41.79 \\
39.65 \\
40.47\end{array}$ & $\begin{array}{l}377.53 \\
378.01 \\
377.30 \\
379.44 \\
378.62\end{array}$ \\
\hline
\end{tabular}


DOE/RL-91-47

Table 3-1. RCRA Water Level Measurement Report 183-H Solar Evaporation Basins - Second Quarter 1991. (sheet 3 of 5)

\begin{tabular}{|c|c|c|c|}
\hline Well & Date & $\begin{array}{c}\text { Depth to water } \\
(\mathrm{ft})\end{array}$ & $\begin{array}{c}\text { Water level elevation } \\
\text { above msl (ft) }\end{array}$ \\
\hline 199-H4-18 & $\begin{array}{l}4 / 10 / 91 \\
4 / 11 / 91 \\
4 / 30 / 91 \\
5 / 16 / 91 \\
5 / 29 / 91 \\
6 / 20 / 91\end{array}$ & $\begin{array}{l}44.72 \\
44.49 \\
44.11 \\
44.66 \\
43.01 \\
43.42\end{array}$ & $\begin{array}{l}377.10 \star \\
377.33 \\
377.71 \\
377.16 \\
378.81 \\
378.40\end{array}$ \\
\hline $199-\mathrm{H} 4-3$ & $\begin{array}{l}4 / 10 / 91 \\
4 / 11 / 91 \\
4 / 30 / 91 \\
5 / 16 / 91 \\
5 / 29 / 91 \\
6 / 07 / 91 \\
6 / 20 / 91\end{array}$ & $\begin{array}{l}43.21 \\
43.00 \\
42.58 \\
43.33 \\
41.27 \\
41.06 \\
41.91\end{array}$ & $\begin{array}{l}377.08 \star \\
377.29 \\
377.71 \\
376.96 \\
379.02 \\
379.23 \star \\
378.38\end{array}$ \\
\hline 199-H4-4 & $\begin{array}{l}4 / 11 / 91 \\
4 / 11 / 91 \\
4 / 30 / 91 \\
5 / 16 / 91 \\
5 / 29 / 91 \\
6 / 06 / 91 \\
6 / 20 / 91\end{array}$ & $\begin{array}{l}35.72 \\
35.59 \\
36.10 \\
35.56 \\
33.73 \\
34.34 \\
37.03\end{array}$ & $\begin{array}{l}377.98 \star \\
378.11 \\
377.60 \\
378.14 \\
379.97 \\
379.36 \star \\
376.67\end{array}$ \\
\hline $199-\mathrm{H} 4-5$ & $\begin{array}{l}4 / 11 / 91 \\
4 / 30 / 91 \\
5 / 16 / 91 \\
5 / 29 / 91 \\
6 / 20 / 91\end{array}$ & $\begin{array}{l}38.72 \\
38.56 \\
39.17 \\
36.42 \\
38.46\end{array}$ & $\begin{array}{l}377.49 \\
377.65 \\
377.04 \\
379.79 \\
377.75\end{array}$ \\
\hline $199-\mathrm{H} 4-6$ & $\begin{array}{l}4 / 11 / 91 \\
4 / 30 / 91 \\
5 / 16 / 91 \\
5 / 29 / 91 \\
6 / 10 / 91 \\
6 / 20 / 91\end{array}$ & $\begin{array}{l}41.83 \\
41.48 \\
41.58 \\
41.13 \\
40.64 \\
40.48\end{array}$ & $\begin{array}{l}377.75 \\
378.10 \\
378.00 \\
378.45 \\
378.94^{\star} \\
379.10\end{array}$ \\
\hline $199-\mathrm{H} 4-7$ & $\begin{array}{l}4 / 11 / 91 \\
4 / 30 / 91 \\
5 / 16 / 91 \\
5 / 29 / 91 \\
6 / 06 / 91 \\
6 / 20 / 91\end{array}$ & $\begin{array}{l}43.16 \\
42.71 \\
43.10 \\
41.95 \\
41.71 \\
41.77\end{array}$ & $\begin{array}{l}377.43 \\
377.88 \\
377.49 \\
378.64 \\
378.88^{\star} \\
378.82\end{array}$ \\
\hline $199-\mathrm{H} 4-8$ & $\begin{array}{l}4 / 11 / 91 \\
4 / 30 / 91 \\
5 / 16 / 91 \\
5 / 29 / 91 \\
6 / 20 / 91\end{array}$ & $\begin{array}{l}42.63 \\
42.14 \\
42.70 \\
41.02 \\
41.23\end{array}$ & $\begin{array}{l}377.37 \\
377.86 \\
377.30 \\
378.98 \\
378.77\end{array}$ \\
\hline
\end{tabular}


DOE/RL-91-47

Table 3-1. RCRA Water Level Measurement Report 183-H Solar Evaporation Basins - Second Quarter 1991. (sheet 4 of 5)

\begin{tabular}{cccc}
\hline We11 & Date & $\begin{array}{c}\text { Depth to water } \\
(\mathrm{ft})\end{array}$ & $\begin{array}{c}\text { Water level elevation } \\
\text { above ms 1 (ft) }\end{array}$ \\
\hline $199-\mathrm{H4}-9$ & $4 / 11 / 91$ & 40.72 & 377.36 \\
& $4 / 30 / 91$ & 40.28 & 377.80 \\
& $5 / 16 / 91$ & 40.96 & 377.12 \\
& $5 / 29 / 91$ & 37.83 & 380.25 \\
& $6 / 06 / 91$ & 38.94 & $379.14 \star$ \\
& $6 / 20 / 91$ & 39.55 & 378.53
\end{tabular}

Wells Monitoring Upper Levels in the Unconfined Aquifer (Ringold Formation)

$\begin{array}{llll}199-H 3-2 C & 4 / 11 / 91 & 40.45 & 377.77 \\ & 4 / 30 / 91 & 40.12 & 378.10 \\ 5 / 16 / 91 & 40.26 & 377.96 \\ 5 / 29 / 91 & 39.63 & 378.59 \\ & 6 / 06 / 91 & 39.39 & 378.83 * \\ 199-H 4-12 C & 6 / 20 / 91 & 39.30 & 378.92 \\ & 4 / 11 / 91 & 35.30 & 378.22 \\ & 4 / 30 / 91 & 36.00 & 377.52 \\ & 5 / 16 / 91 & 35.87 & 377.65 \\ & 5 / 29 / 91 & 34.16 & 379.36 \\ 199-H 4-15 C S & 6 / 07 / 91 & 33.51 & 380.01 * \\ & 6 / 20 / 91 & 36.66 & 376.86 \\ & 4 / 11 / 91 & 29.08 & 378.36 \\ & 4 / 30 / 91 & 29.75 & 377.69 \\ & 5 / 16 / 91 & 29.59 & 377.85 \\ & 5 / 29 / 91 & 27.82 & 379.62 \\ & 6 / 20 / 91 & 30.54 & 376.90\end{array}$

Well Monitoring Mid-Level in the Unconfined Aquifer (Ringold Formation)

$\begin{array}{llll}199-H 4-15 C R & 4 / 11 / 91 & 31.58 & 375.79 \\ & 4 / 30 / 91 & 31.43 & 375.94 \\ 5 / 16 / 91 & 31.28 & 376.09 \\ & 5 / 29 / 91 & 30.84 & 376.53 \\ & 6 / 20 / 91 & 30.33 & 377.04\end{array}$


$\mathrm{DOE} / \mathrm{RL}-91-47$

Table 3-1. RCRA Water Level Measurement Report 183-H Solar Evaporation Basins - Second Quarter 1991. (sheet 5 of 5)

\begin{tabular}{lccc} 
Wel1 & Date & $\begin{array}{c}\text { Depth to water } \\
(\mathrm{ft})\end{array}$ & $\begin{array}{c}\text { Water level elevation } \\
\text { above ms 1 (ft) }\end{array}$ \\
\hline Wel1 Monitoring the Bottom of the Unconfined Aquifer (Ringold Formation) \\
199-H4-15CQ & $4 / 11 / 91$ & 12.15 & 395.12 \\
& $4 / 30 / 91$ & 12.13 & 395.14 \\
& $5 / 16 / 91$ & 12.48 & 394.79 \\
& $5 / 29 / 91$ & 11.55 & 395.72 \\
& $6 / 20 / 91$ & 12.78 & 394.49 \\
\hline
\end{tabular}

NOTES: 1. Water level elevations are calculated by subtracting the measured depth to water from the surveyed elevation for the well.

2. Depth-to-water values are transcribed from field records.

3. Elevations marked with an ' $*$ ' were measured at the time of sampling. 


\subsection{AREAS LOW-LEVEL BURIAL GROUNDS}

R. B. Mercer

\section{Westinghouse Hanford Company}

The groundwater monitoring network for the Low-Level Burial Grounds, consisting of five Low-Level Waste Management Areas (LLWMA), has not changed from that described in the quarterly report for the period July 1 through September 30, 1990 (DOE-RL 1990). Please refer to that report for a description of the facilities and the monitoring networks.

Water level measurements for the second quarter of 1991 are presented in Table 4-1. 
Table 4-1. RCRA Water Level Measurement Report Low-Level Burial Grounds - Second Quarter 1991. (sheet 1 of 4)

\begin{tabular}{|c|c|c|c|}
\hline Well & Date & $\begin{array}{c}\text { Depth to water } \\
(\mathrm{ft})\end{array}$ & $\begin{array}{l}\text { Water level elevation } \\
\text { above msl ( } \mathrm{ft} \text { ) }\end{array}$ \\
\hline \multicolumn{4}{|c|}{ LLWMA-1 Wells Monitoring the Top of the Unconfined Aquifer } \\
\hline 299-E28-26 & $\begin{array}{l}4 / 19 / 91 \\
5 / 24 / 91 \\
6 / 12 / 91\end{array}$ & $\begin{array}{l}283.95 \\
283.99 \\
283.98\end{array}$ & $\begin{array}{l}403.31 \\
403.27 \\
403.28\end{array}$ \\
\hline 299-E28-27 & $\begin{array}{l}4 / 19 / 91 \\
5 / 24 / 91 \\
6 / 12 / 91\end{array}$ & $\begin{array}{l}277.16 \\
277.36 \\
277.20\end{array}$ & $\begin{array}{l}403.21 \\
403.01 \\
403.17\end{array}$ \\
\hline 299-E28-28 & $\begin{array}{l}4 / 19 / 91 \\
5 / 24 / 91 \\
6 / 12 / 91\end{array}$ & $\begin{array}{l}283.41 \\
283.42 \\
283.45\end{array}$ & $\begin{array}{l}403.14 \\
403.13 \\
403.10\end{array}$ \\
\hline 299-E32-2 & $\begin{array}{l}4 / 19 / 91 \\
5 / 24 / 91 \\
6 / 12 / 91\end{array}$ & $\begin{array}{l}266.95 \\
266.98 \\
266.99\end{array}$ & $\begin{array}{l}403.11 \\
403.08 \\
403.07\end{array}$ \\
\hline 299-E32-3 & $\begin{array}{l}4 / 19 / 91 \\
5 / 24 / 91 \\
6 / 12 / 91\end{array}$ & $\begin{array}{l}273.34 \\
273.35 \\
273.38\end{array}$ & $\begin{array}{l}403.17 \\
403.16 \\
403.13\end{array}$ \\
\hline 299-E32-4 & $\begin{array}{l}4 / 19 / 91 \\
5 / 24 / 91 \\
6 / 12 / 91\end{array}$ & $\begin{array}{l}282.84 \\
282.83 \\
282.83\end{array}$ & $\begin{array}{l}403.04 \\
403.05 \\
403.05\end{array}$ \\
\hline $299-E 32-5$ & $\begin{array}{l}4 / 19 / 91 \\
5 / 24 / 91 \\
6 / 12 / 91\end{array}$ & $\begin{array}{l}278.98 \\
278.98 \\
279.01\end{array}$ & $\begin{array}{l}403.16 \\
403.16 \\
403.13\end{array}$ \\
\hline $299-E 33-28$ & $\begin{array}{l}4 / 19 / 91 \\
5 / 24 / 91 \\
6 / 12 / 91\end{array}$ & $\begin{array}{l}260.94 \\
260.98 \\
260.99\end{array}$ & $\begin{array}{l}403.29 \\
403.25 \\
403.24\end{array}$ \\
\hline 299-E33-29 & $\begin{array}{l}4 / 19 / 91 \\
5 / 24 / 91 \\
6 / 12 / 91\end{array}$ & $\begin{array}{l}270.49 \\
270.52 \\
270.55\end{array}$ & $\begin{array}{l}403.28 \\
403.25 \\
403.22\end{array}$ \\
\hline 299-E33-30 & $\begin{array}{l}4 / 19 / 91 \\
5 / 24 / 91 \\
6 / 12 / 91\end{array}$ & $\begin{array}{l}260.44 \\
260.47 \\
260.47\end{array}$ & $\begin{array}{l}403.26 \\
403.23 \\
403.23\end{array}$ \\
\hline 299-E33-34 & $\begin{array}{l}4 / 19 / 91 \\
5 / 24 / 91 \\
6 / 12 / 91\end{array}$ & $\begin{array}{l}230.14 \\
230.17 \\
230.19\end{array}$ & $\begin{array}{l}403.19 \\
403.16 \\
403.14\end{array}$ \\
\hline 299-E33-35 & $\begin{array}{l}4 / 19 / 91 \\
5 / 24 / 91 \\
6 / 12 / 91\end{array}$ & $\begin{array}{l}239.85 \\
239.86 \\
239.89\end{array}$ & $\begin{array}{l}403.16 \\
403.15 \\
403.12\end{array}$ \\
\hline
\end{tabular}


Table 4-1. RCRA Water Level Measurement Report Low-Level Burial Grounds - Second Quarter 1991. (sheet 2 of 4)

\begin{tabular}{cccc}
\hline We11 & Date & $\begin{array}{c}\text { Depth to water } \\
(\mathrm{ft})\end{array}$ & $\begin{array}{c}\text { Water level ele } \\
\text { above ms } 1 \text { ( }\end{array}$ \\
\hline LLWMA-2 & We1ls Monitoring the Top of the Unconfined Aquifer \\
$299-E 27-10$ & $6 / 06 / 91$ & 220.74 & 403.73 \\
$299-E 27-11$ & $6 / 06 / 91$ & 240.13 & 403.16 \\
$299-E 27-8$ & $6 / 06 / 91$ & 234.58 & 403.25 \\
$299-E 27-9$ & $6 / 06 / 91$ & 225.72 & 403.49 \\
$299-E 34-2$ & $4 / 23 / 91$ & 227.31 & 403.49 \\
& $5 / 20 / 91$ & 227.43 & 403.37 \\
$299-E 34-3$ & $6 / 18 / 91$ & 227.36 & 403.44 \\
$299-E 34-5$ & $6 / 06 / 91$ & 207.56 & 403.96 \\
& $4 / 23 / 91$ & 186.43 & 404.36 \\
$299-E 34-6$ & $5 / 20 / 91$ & 186.53 & 404.26 \\
$299-E 34-7$ & $6 / 18 / 91$ & 186.53 & 404.26 \\
$299-E 35-1$ & $6 / 06 / 91$ & 194.54 & 403.29 \\
& $6 / 06 / 91$ & 200.80 & 403.47
\end{tabular}

LLWMA-3 Wells Monitoring the Top of the Unconfined Aquifer

$\begin{array}{llll}299-W 10-13 & 4 / 19 / 91 & 234.32 & 464.72 \\ & 5 / 24 / 91 & 234.41 & 464.63 \\ 299-W 6-2 & 6 / 14 / 91 & 234.62 & 464.42 \\ & 4 / 19 / 91 & 232.42 & 460.03 \\ & 5 / 24 / 91 & 232.54 & 459.91 \\ 299-W 7-1 & 6 / 14 / 91 & 232.71 & 459.74 \\ & 4 / 19 / 91 & 230.36 & 460.35 \\ & 5 / 24 / 91 & 230.47 & 460.24 \\ 299-W 7-10 & 6 / 14 / 91 & 230.63 & 460.08 \\ & 4 / 19 / 91 & 230.33 & 459.33 \\ 299-W 7-2 & 5 / 24 / 91 & 230.46 & 459.20 \\ & 6 / 14 / 91 & 230.62 & 459.04 \\ & 4 / 19 / 91 & 216.24 & 459.35 \\ 299-W 7-4 & 5 / 24 / 91 & 216.34 & 459.25 \\ & 6 / 14 / 91 & 216.50 & 459.09 \\ & 4 / 19 / 91 & 210.26 & 461.43 \\ & 5 / 24 / 91 & 210.39 & 461.30 \\ & 6 / 14 / 91 & 210.53 & 461.16\end{array}$


Table 4-1. RCRA Water Level Measurement Report Low-Level Burial Grounds - Second Quarter 1991. (sheet 3 of 4)

\begin{tabular}{cccc}
\hline We11 & Date & $\begin{array}{c}\text { Depth to water } \\
(\mathrm{ft})\end{array}$ & $\begin{array}{c}\text { Water level elevation } \\
\text { above ms } 1(\mathrm{ft})\end{array}$ \\
\hline $299-W 7-5$ & $4 / 19 / 91$ & 214.16 & 458.89 \\
& $5 / 24 / 91$ & 214.26 & 458.79 \\
$299-W 7-6$ & $6 / 14 / 91$ & 214.41 & 458.64 \\
& $4 / 19 / 91$ & 219.82 & 458.82 \\
& $5 / 24 / 91$ & 219.98 & 458.65 \\
$299-W 7-7$ & $6 / 14 / 91$ & 220.11 & 458.53 \\
& $4 / 19 / 91$ & 215.95 & 458.99 \\
$299-W 7-8$ & $5 / 24 / 91$ & 216.09 & 458.85 \\
& $6 / 14 / 91$ & 216.24 & 458.70 \\
& $4 / 19 / 91$ & 229.71 & 457.64 \\
$299-W 7-9$ & $5 / 24 / 91$ & 229.82 & 457.53 \\
& $6 / 14 / 91$ & 229.95 & 457.40 \\
& $4 / 19 / 91$ & 231.35 & 460.74 \\
$299-W 8-1$ & $5 / 24 / 91$ & 231.46 & 460.63 \\
& $6 / 14 / 91$ & 231.60 & 460.49 \\
& $4 / 19 / 91$ & 240.56 & 460.77 \\
$299-W 9-1$ & $5 / 24 / 91$ & 240.68 & 460.65 \\
& $6 / 14 / 91$ & 240.84 & 460.49 \\
& $4 / 19 / 91$ & 274.19 & 463.54 \\
& $5 / 24 / 91$ & 274.33 & 463.40 \\
& $6 / 14 / 91$ & 274.55 & 463.18
\end{tabular}

LLWMA-3 Wells Monitoring the Bottom of the Unconfined Aquifer

$\begin{array}{llll}299-W 10-14 & 4 / 19 / 91 & 235.16 & 464.27 \\ & 5 / 24 / 91 & 235.28 & 464.15 \\ & 6 / 14 / 91 & 235.45 & 463.98 \\ 299-W 7-3 & 4 / 19 / 91 & 217.95 & 458.19 \\ & 5 / 24 / 91 & 218.10 & 458.04 \\ & 6 / 14 / 91 & 218.25 & 457.89\end{array}$

LLWMA-4 Wells Monitoring the Top of the Unconfined Aquifer

$\begin{array}{llll}299-W 15-15 & 6 / 07 / 91 & 231.44 & 466.52 \\ 299-W 15-16 & 6 / 07 / 91 & 217.64 & 467.25 \\ 299-W 15-18 & 6 / 07 / 91 & 218.08 & 467.63 \\ 299-W 15-19 & 6 / 07 / 91 & 225.34 & 466.26 \\ 299-W 15-20 & 6 / 07 / 91 & 232.28 & 466.08\end{array}$




$$
\mathrm{DOE} / \mathrm{RL}-91-47
$$

Table 4-1. RCRA Water Level Measurement Report Low-Level Burial Grounds - Second Quarter 1991. (sheet 4 of 4)

\begin{tabular}{cccc}
\hline We11 & Date & $\begin{array}{c}\text { Depth to water } \\
(\mathrm{ft})\end{array}$ & $\begin{array}{c}\text { Water Tevel elevation } \\
\text { above ms }(\mathrm{ft})\end{array}$ \\
\hline $299-W 15-23$ & $6 / 07 / 91$ & 233.19 & 466.30 \\
$299-W 15-24$ & $6 / 07 / 91$ & 232.63 & 466.74 \\
$299-W 18-21$ & $6 / 07 / 91$ & 201.81 & 466.81 \\
$299-W 18-23$ & $6 / 07 / 91$ & 230.04 & 466.77 \\
$299-W 18-24$ & $6 / 07 / 91$ & 216.64 & 467.71 \\
$299-W 18-26$ & $6 / 07 / 91$ & 232.27 & 466.78
\end{tabular}

LLWMA-4 Wells Monitoring the Bottom of the Unconfined Aquifer

$\begin{array}{llll}299-W 15-17 & 6 / 07 / 91 & 217.76 & 466.88 \\ 299-W 18-22 & 6 / 07 / 91 & 202.44 & 466.05\end{array}$

NOTES: 1. Water level elevations are calculated by subtracting the measured depth to water from the surveyed elevation for the well.

2. Depth-to-water values are transcribed from field records. 
$\mathrm{DOE} / \mathrm{RL}-91-47$

This page intentionally left blank. 


\subsection{0-N AREA RCRA SITES}

M. J. Hartman

Westinghouse Hanford Company

The 1301-N and 1324-N/NA Facilities were monitored under a groundwater quality assessment program during the past quarter (April 1 through June 30 , 1991). The 1325-N site remained in a detection-level program.

Well N-57 has been substituted for well N-6 as an upgradient well to better reflect the influence of the 1324-N/NA facilities on groundwater quality (Hartman 1990).

Downgradient wells $N-58, N-60$, and $N-61$ at 1324-N/NA have too little water to sample. New wells will be installed (Hartman 1991).

Wells $\mathrm{N}-36$ and $\mathrm{N}-39$, formerly monitored as downgradient wells for $1325-\mathrm{N}$, have too little water to sample.

Water levels are measured monthly in most of the wells in the 100-N Area. Water levels for April, May, and June are presented in Table 5-1.

The wells in the 100-N Area networks were scheduled for sampling in June. However, because of logistical problems, only one well was sampled (N-57, sampled for the 1301-N site). Results of analyses had not been received by the end of the quarter and will be included in a future report. 
Table 5-1. RCRA Water Level Measurement Report 100-N Area - Second Quarter 1991. (sheet 1 of 5)

\begin{tabular}{|c|c|c|c|}
\hline We11 & Date & $\begin{array}{c}\text { Depth to water } \\
(\mathrm{ft})\end{array}$ & $\begin{array}{c}\text { Water level elevation } \\
\text { above msl (ft) }\end{array}$ \\
\hline \multicolumn{4}{|c|}{ Wells Monitoring the Top of the Unconfined Aquifer } \\
\hline $199-N-14$ & $\begin{array}{l}4 / 25 / 91 \\
5 / 30 / 91 \\
6 / 24 / 91\end{array}$ & $\begin{array}{l}64.86 \\
63.97 \\
65.14\end{array}$ & $\begin{array}{l}388.29 \\
389.18 \\
388.01\end{array}$ \\
\hline $199-N-16$ & $\begin{array}{l}4 / 25 / 91 \\
5 / 30 / 91 \\
6 / 24 / 91\end{array}$ & $\begin{array}{l}66.63 \\
66.35 \\
66.23\end{array}$ & $\begin{array}{l}390.07 \\
390.35 \\
390.47\end{array}$ \\
\hline $199-N-17$ & $\begin{array}{l}4 / 25 / 91 \\
5 / 30 / 91 \\
6 / 24 / 91\end{array}$ & $\begin{array}{l}72.06 \\
71.36 \\
71.99\end{array}$ & $\begin{array}{l}389.14 \\
389.84 \\
389.21\end{array}$ \\
\hline $199-N-18$ & $\begin{array}{l}4 / 25 / 91 \\
5 / 30 / 91 \\
6 / 24 / 91\end{array}$ & $\begin{array}{l}69.51 \\
68.62 \\
69.98\end{array}$ & $\begin{array}{l}388.99 \\
389.88 \\
388.52\end{array}$ \\
\hline $199-N-19$ & $\begin{array}{l}4 / 25 / 91 \\
5 / 30 / 91 \\
6 / 24 / 91\end{array}$ & $\begin{array}{l}64.73 \\
63.77 \\
65.73\end{array}$ & $\begin{array}{l}389.17 \\
390.13 \\
388.17\end{array}$ \\
\hline $199-N-2$ & $\begin{array}{l}4 / 26 / 91 \\
5 / 30 / 91 \\
6 / 25 / 91\end{array}$ & $\begin{array}{l}70.49 \\
69.59 \\
70.36\end{array}$ & $\begin{array}{l}389.34 \\
390.24 \\
389.47\end{array}$ \\
\hline $199-N-20$ & $\begin{array}{l}4 / 25 / 91 \\
5 / 30 / 91 \\
6 / 24 / 91\end{array}$ & $\begin{array}{l}66.45 \\
65.64 \\
67.16\end{array}$ & $\begin{array}{l}389.45 \\
390.26 \\
388.74\end{array}$ \\
\hline $199-N-21$ & $\begin{array}{l}4 / 25 / 91 \\
5 / 30 / 91 \\
6 / 24 / 91\end{array}$ & $\begin{array}{l}67.56 \\
66.62 \\
68.31\end{array}$ & $\begin{array}{l}389.44 \\
390.38 \\
388.69\end{array}$ \\
\hline $199-N-23$ & $\begin{array}{l}4 / 25 / 91 \\
5 / 30 / 91 \\
6 / 24 / 91\end{array}$ & $\begin{array}{l}67.01 \\
65.89 \\
67.96\end{array}$ & $\begin{array}{l}389.29 \\
390.41 \\
388.34\end{array}$ \\
\hline $199-N-25$ & $\begin{array}{l}4 / 25 / 91 \\
5 / 30 / 91 \\
6 / 24 / 91\end{array}$ & $\begin{array}{l}36.48 \\
34.83 \\
38.85\end{array}$ & $\begin{array}{l}389.32 \\
390.97 \\
386.95\end{array}$ \\
\hline $199-N-26$ & $\begin{array}{l}4 / 25 / 91 \\
5 / 30 / 91 \\
6 / 24 / 91\end{array}$ & $\begin{array}{l}66.44 \\
65.11 \\
68.05\end{array}$ & $\begin{array}{l}389.36 \\
390.69 \\
387.75\end{array}$ \\
\hline $199-N-27$ & $\begin{array}{l}4 / 03 / 91 \\
4 / 25 / 91 \\
5 / 09 / 91 \\
5 / 30 / 91 \\
6 / 24 / 91\end{array}$ & $\begin{array}{l}59.11 \\
59.10 \\
59.01 \\
59.03 \\
58.96\end{array}$ & $\begin{array}{l}389.97 \\
389.98 \\
390.07 \\
390.05 \\
390.12\end{array}$ \\
\hline
\end{tabular}


Table 5-1. RCRA Water Level Measurement Report 100-N Area - Second Quarter 1991. (sheet 2 of 5)

\begin{tabular}{|c|c|c|c|}
\hline Well & Date & $\begin{array}{c}\text { Depth to water } \\
(f t)\end{array}$ & $\begin{array}{c}\text { Water level elevation } \\
\text { above msl (ft) }\end{array}$ \\
\hline $199-N-28$ & $\begin{array}{l}4 / 25 / 91 \\
5 / 30 / 91 \\
6 / 24 / 91\end{array}$ & $\begin{array}{l}73.84 \\
73.86 \\
73.71\end{array}$ & $\begin{array}{l}390.40 \\
390.38 \\
390.53\end{array}$ \\
\hline $199-N-29$ & $\begin{array}{l}4 / 25 / 91 \\
5 / 30 / 91 \\
6 / 24 / 91\end{array}$ & $\begin{array}{l}74.88 \\
74.89 \\
74.69\end{array}$ & $\begin{array}{l}390.37 \\
390.36 \\
390.56\end{array}$ \\
\hline $199-N-3$ & $\begin{array}{l}4 / 26 / 91 \\
5 / 30 / 91 \\
6 / 25 / 91\end{array}$ & $\begin{array}{l}70.08 \\
69.05 \\
70.16\end{array}$ & $\begin{array}{l}389.37 \\
390.40 \\
389.29\end{array}$ \\
\hline $199-N-31$ & $\begin{array}{l}4 / 25 / 91 \\
5 / 30 / 91 \\
6 / 24 / 91\end{array}$ & $\begin{array}{l}72.93 \\
72.89 \\
72.58\end{array}$ & $\begin{array}{l}389.70 \\
389.74 \\
390.05\end{array}$ \\
\hline $199-N-32$ & $\begin{array}{l}4 / 26 / 91 \\
5 / 30 / 91 \\
6 / 24 / 91\end{array}$ & $\begin{array}{l}72.30 \\
72.21 \\
71.92\end{array}$ & $\begin{array}{l}389.78 \\
389.87 \\
390.16\end{array}$ \\
\hline $199-N-33$ & $\begin{array}{l}4 / 25 / 91 \\
5 / 30 / 91 \\
6 / 24 / 91\end{array}$ & $\begin{array}{l}70.76 \\
70.13 \\
69.70\end{array}$ & $\begin{array}{l}389.11 \\
389.74 \\
390.17\end{array}$ \\
\hline $199-N-34$ & $\begin{array}{l}4 / 03 / 91 \\
4 / 25 / 91 \\
5 / 09 / 91 \\
5 / 30 / 91 \\
6 / 24 / 91\end{array}$ & $\begin{array}{l}69.69 \\
69.80 \\
69.60 \\
69.67 \\
69.48\end{array}$ & $\begin{array}{l}389.94 \\
389.83 \\
390.03 \\
389.96 \\
390.15\end{array}$ \\
\hline $199-N-37$ & $\begin{array}{l}4 / 26 / 91 \\
5 / 30 / 91 \\
6 / 24 / 91\end{array}$ & $\begin{array}{l}66.80 \\
66.68 \\
66.35\end{array}$ & $\begin{array}{l}389.32 \\
389.44 \\
389.77\end{array}$ \\
\hline $199-N-39$ & $\begin{array}{l}4 / 26 / 91 \\
5 / 30 / 91 \\
6 / 24 / 91\end{array}$ & $\begin{array}{l}65.33 \\
65.18 \\
64.71\end{array}$ & $\begin{array}{l}388.98 \\
389.13 \\
389.60\end{array}$ \\
\hline $199-N-4$ & $\begin{array}{l}4 / 26 / 91 \\
5 / 30 / 91 \\
6 / 25 / 91\end{array}$ & $\begin{array}{l}69.12 \\
68.92 \\
68.66\end{array}$ & $\begin{array}{l}389.61 \\
389.81 \\
390.07\end{array}$ \\
\hline $199-N-40$ & $\begin{array}{l}4 / 26 / 91 \\
5 / 30 / 91 \\
6 / 24 / 91\end{array}$ & $\begin{array}{l}67.65 \\
67.45 \\
67.02\end{array}$ & $\begin{array}{l}388.70 \\
388.90 \\
389.33\end{array}$ \\
\hline $199-N-41$ & $\begin{array}{l}4 / 26 / 91 \\
5 / 30 / 91 \\
6 / 24 / 91\end{array}$ & $\begin{array}{l}69.38 \\
68.88 \\
68.85\end{array}$ & $\begin{array}{l}388.21 \\
388.71 \\
388.74\end{array}$ \\
\hline
\end{tabular}


Table 5-1. RCRA Water Level Measurement Report 100-N Area - Second Quarter 1991. (sheet 3 of 5)

\begin{tabular}{|c|c|c|c|}
\hline Wel1 & Date & $\begin{array}{c}\text { Depth to water } \\
(\mathrm{ft})\end{array}$ & $\begin{array}{c}\text { Water level elevation } \\
\text { above msl (ft) }\end{array}$ \\
\hline $199-N-42$ & $\begin{array}{l}4 / 25 / 91 \\
5 / 30 / 91 \\
6 / 24 / 91\end{array}$ & $\begin{array}{l}66.58 \\
66.18 \\
66.11\end{array}$ & $\begin{array}{l}388.56 \\
388.96 \\
389.03\end{array}$ \\
\hline $199-N-44$ & $\begin{array}{l}4 / 25 / 91 \\
5 / 30 / 91 \\
6 / 24 / 91\end{array}$ & $\begin{array}{l}71.74 \\
71.63 \\
70.92\end{array}$ & $\begin{array}{l}388.96 \\
389.07 \\
389.78\end{array}$ \\
\hline $199-N-49$ & $\begin{array}{l}4 / 25 / 91 \\
5 / 30 / 91 \\
6 / 24 / 91\end{array}$ & $\begin{array}{l}62.38 \\
62.07 \\
61.72\end{array}$ & $\begin{array}{l}388.34 \\
388.65 \\
389.00\end{array}$ \\
\hline $199-N-50$ & $\begin{array}{l}4 / 25 / 91 \\
5 / 30 / 91 \\
6 / 24 / 91\end{array}$ & $\begin{array}{l}75.31 \\
74.26 \\
75.94\end{array}$ & $\begin{array}{l}378.05 \\
379.10 \\
377.42\end{array}$ \\
\hline $199-N-51$ & $\begin{array}{l}4 / 25 / 91 \\
5 / 30 / 91 \\
6 / 24 / 91\end{array}$ & $\begin{array}{l}73.98 \\
72.32 \\
75.65\end{array}$ & $\begin{array}{l}388.20 \\
389.86 \\
386.53\end{array}$ \\
\hline $199-N-52$ & $\begin{array}{l}4 / 25 / 91 \\
5 / 30 / 91 \\
6 / 24 / 91\end{array}$ & $\begin{array}{l}73.22 \\
73.22 \\
73.04\end{array}$ & $\begin{array}{l}390.48 \\
390.48 \\
390.66\end{array}$ \\
\hline $199-N-54$ & $\begin{array}{l}4 / 25 / 91 \\
5 / 30 / 91 \\
6 / 24 / 91\end{array}$ & $\begin{array}{l}67.83 \\
67.54 \\
67.35\end{array}$ & $\begin{array}{l}389.68 \\
389.97 \\
390.16\end{array}$ \\
\hline $199-N-55$ & $\begin{array}{l}4 / 25 / 91 \\
5 / 30 / 91 \\
6 / 24 / 91\end{array}$ & $\begin{array}{l}68.17 \\
67.88 \\
67.70\end{array}$ & $\begin{array}{l}389.68 \\
389.97 \\
390.15\end{array}$ \\
\hline $199-N-56$ & $\begin{array}{l}4 / 25 / 91 \\
5 / 30 / 91 \\
6 / 24 / 91\end{array}$ & $\begin{array}{l}68.84 \\
68.37 \\
68.33\end{array}$ & $\begin{array}{l}389.25 \\
389.72 \\
389.76\end{array}$ \\
\hline $199-N-57$ & $\begin{array}{l}4 / 25 / 91 \\
5 / 30 / 91 \\
6 / 24 / 91 \\
6 / 28 / 91\end{array}$ & $\begin{array}{l}67.53 \\
67.41 \\
67.15 \\
67.20\end{array}$ & $\begin{array}{c}390.23 \\
390.35 \\
390.61 \\
390.56^{\star}\end{array}$ \\
\hline $199-N-59$ & $\begin{array}{l}4 / 25 / 91 \\
5 / 30 / 91 \\
6 / 24 / 91\end{array}$ & $\begin{array}{l}68.57 \\
68.40 \\
68.04\end{array}$ & $\begin{array}{l}390.96 \\
391.13 \\
391.49\end{array}$ \\
\hline $199-N-6$ & $\begin{array}{l}4 / 26 / 91 \\
5 / 30 / 91 \\
6 / 25 / 91\end{array}$ & $\begin{array}{l}70.53 \\
71.99 \\
70.10\end{array}$ & $\begin{array}{l}390.44 \\
388.98 \\
390.87\end{array}$ \\
\hline
\end{tabular}


Table 5-1. RCRA Water Level Measurement Report 100-N Area - Second Quarter 1991. (sheet 4 of 5)

\begin{tabular}{|c|c|c|c|}
\hline Well & Date & $\begin{array}{c}\text { Depth to water } \\
(\mathrm{ft})\end{array}$ & $\begin{array}{c}\text { Water level elevation } \\
\text { above } \mathrm{msl}(\mathrm{ft})\end{array}$ \\
\hline $199-N-60$ & $\begin{array}{l}4 / 25 / 91 \\
5 / 30 / 91 \\
6 / 24 / 91\end{array}$ & $\begin{array}{l}71.02 \\
70.45 \\
70.08\end{array}$ & $\begin{array}{l}390.92 \\
391.49 \\
391.86\end{array}$ \\
\hline $199-N-62$ & $\begin{array}{l}4 / 25 / 91 \\
5 / 30 / 91 \\
6 / 24 / 91\end{array}$ & $\begin{array}{l}73.07 \\
73.08 \\
72.93\end{array}$ & $\begin{array}{l}390.52 \\
390.51 \\
390.66\end{array}$ \\
\hline $199-N-63$ & $\begin{array}{l}4 / 25 / 91 \\
5 / 30 / 91 \\
6 / 24 / 91\end{array}$ & $\begin{array}{l}76.32 \\
76.33 \\
76.02\end{array}$ & $\begin{array}{l}390.38 \\
390.37 \\
390.68\end{array}$ \\
\hline $199-N-64$ & $\begin{array}{l}4 / 26 / 91 \\
5 / 30 / 91 \\
6 / 25 / 91\end{array}$ & $\begin{array}{l}64.49 \\
64.38 \\
64.00\end{array}$ & $\begin{array}{l}390.14 \\
390.25 \\
390.63\end{array}$ \\
\hline $199-N-65$ & $\begin{array}{l}4 / 26 / 91 \\
5 / 30 / 91 \\
6 / 25 / 91\end{array}$ & $\begin{array}{l}66.59 \\
66.45 \\
66.14\end{array}$ & $\begin{array}{l}389.85 \\
389.99 \\
390.30\end{array}$ \\
\hline $199-N-66$ & $\begin{array}{l}4 / 26 / 91 \\
5 / 30 / 91 \\
6 / 25 / 91\end{array}$ & $\begin{array}{l}75.70 \\
75.48 \\
75.20\end{array}$ & $\begin{array}{l}389.55 \\
389.77 \\
390.05\end{array}$ \\
\hline $199-N-67$ & $\begin{array}{l}4 / 26 / 91 \\
5 / 30 / 91 \\
6 / 25 / 91\end{array}$ & $\begin{array}{l}69.57 \\
68.77 \\
69.29\end{array}$ & $\begin{array}{l}388.89 \\
389.69 \\
389.17\end{array}$ \\
\hline $199-N-8 S$ & $\begin{array}{l}4 / 11 / 91 \\
4 / 25 / 91 \\
5 / 09 / 91 \\
6 / 25 / 91\end{array}$ & $\begin{array}{l}15.26 \\
15.67 \\
16.10 \\
18.04\end{array}$ & $\begin{array}{l}389.31 \\
388.90 \\
388.47 \\
386.53\end{array}$ \\
\hline $699-81-58$ & $\begin{array}{l}4 / 26 / 91 \\
5 / 30 / 91 \\
6 / 24 / 91\end{array}$ & $\begin{array}{l}44.64 \\
44.78 \\
44.85\end{array}$ & $\begin{array}{l}394.91 \\
394.77 \\
394.70\end{array}$ \\
\hline
\end{tabular}

Wells Monitoring the Bottom of the Unconfined Aquifer $199-N-69$

$\begin{array}{ll}4 / 26 / 91 & 69.94 \\ 5 / 30 / 91 & 69.17 \\ 6 / 25 / 91 & 69.77\end{array}$

388.90 389.67 389.07 
$\mathrm{DOE} / \mathrm{RL}-91-47$

Table 5-1. RCRA Water Level Measurement Report 100-N Area - Second Quarter 1991. (sheet 5 of 5)

\begin{tabular}{|c|c|c|c|}
\hline Well & Date & $\begin{array}{c}\text { Depth to water } \\
(\mathrm{ft})\end{array}$ & $\begin{array}{l}\text { Water level elevation } \\
\text { above msl (ft) }\end{array}$ \\
\hline \multicolumn{4}{|c|}{ Wells Monitoring the Bottom of the Unconfined Aquifer } \\
\hline $199-N-70$ & $\begin{array}{l}4 / 26 / 91 \\
5 / 30 / 91 \\
6 / 24 / 91\end{array}$ & $\begin{array}{l}65.22 \\
65.05 \\
64.63\end{array}$ & $\begin{array}{r}388.99 \\
389.16 \\
389.58\end{array}$ \\
\hline $\begin{array}{l}\text { NOTES: } 1 \\
\qquad \begin{array}{l}2 . \\
3\end{array}\end{array}$ & \multicolumn{3}{|c|}{$\begin{array}{l}\text { Water level elevations are calculated by subtracting the } \\
\text { measured depth to water from the surveyed elevation for the } \\
\text { well. } \\
\text { Depth-to-water values are transcribed from field records. } \\
\text { Elevations marked with an } 1 * 1 \text { were measured at the time of } \\
\text { sampling. }\end{array}$} \\
\hline
\end{tabular}




\section{$6.0 \quad 216-A-29$ DITCH}

\section{G. L. Kasza \\ Westinghouse Hanford Company}

The 216-A-29 Ditch currently is in groundwater quality assessment monitoring. The 216-A-29 groundwater quality assessment monitoring activities and expanded monitoring well network are described in a previous quarterly report (DOE-RL 1990).

The groundwater monitoring network for the 216-A-29 Ditch is in compliance with the applicable regulations since the release of the Ground Water Monitoring Plan for the 216-A-29 Ditch (Kasza 1991). In accordance with the plan, two additional RCRA-compliant we?ls (699-43-43 and 699-43-45) have been added to the network to act as the groundwater monitoring network upgradient wells.

Water levels are measured monthly in the wells comprising the 216-A-29 Ditch monitoring network and quarterly for the surrounding wells that provide supplemental data for the groundwater quality assessment investigation. Water level measurements obtained during this quarter are presented in Table 6-1.

No wells in the detection monitoring network were sampled during the report period. One of the supplemental wells for the assessment program was sampled in late June. No analytical information was returned in time for inclusion in this report. 
Table 6-1. RCRA Water Level Measurement Report 216-A-29 Ditch - Second Quarter 1991. (sheet 1 of 2)

\begin{tabular}{|c|c|c|c|}
\hline Well & Date & $\begin{array}{c}\text { Depth to water } \\
\text { (ft) }\end{array}$ & $\begin{array}{c}\text { Water level elevation } \\
\text { above } \mathrm{msl}(\mathrm{ft})\end{array}$ \\
\hline \multicolumn{4}{|c|}{ Wells in the Detection Monitoring System } \\
\hline $299-E 25-26$ & $\begin{array}{l}4 / 17 / 91 \\
4 / 18 / 91 \\
5 / 24 / 91 \\
6 / 13 / 91\end{array}$ & $\begin{array}{l}264.60 \\
264.54 \\
264.62 \\
264.58\end{array}$ & $\begin{array}{l}403.92^{\star} \\
403.98 \\
403.90 \\
403.94\end{array}$ \\
\hline $299-E 25-28$ & $\begin{array}{l}4 / 10 / 91 \\
4 / 17 / 91 \\
4 / 18 / 91 \\
5 / 24 / 91 \\
6 / 13 / 91\end{array}$ & $\begin{array}{l}258.62 \\
258.25 \\
258.37 \\
258.38 \\
258.44\end{array}$ & $\begin{array}{l}403.82 * \\
404.19 * \\
404.07 \\
404.06 \\
404.00\end{array}$ \\
\hline $299-E 25-32 P$ & $\begin{array}{l}4 / 11 / 91 \\
4 / 18 / 91 \\
5 / 24 / 91 \\
6 / 13 / 91\end{array}$ & $\begin{array}{l}266.15 \\
266.17 \\
266.04 \\
266.12\end{array}$ & $\begin{array}{l}403.89 \star \\
403.87 \\
404.00 \\
403.92\end{array}$ \\
\hline $299-E 25-34$ & $\begin{array}{l}4 / 10 / 91 \\
4 / 18 / 91 \\
5 / 24 / 91 \\
6 / 13 / 91\end{array}$ & $\begin{array}{l}258.92 \\
258.90 \\
258.90 \\
258.98\end{array}$ & $\begin{array}{l}403.95^{\star} \\
403.97 \\
403.97 \\
403.89\end{array}$ \\
\hline $299-E 25-35$ & $\begin{array}{l}4 / 17 / 91 \\
4 / 18 / 91 \\
5 / 24 / 91 \\
6 / 13 / 91\end{array}$ & $\begin{array}{l}270.78 \\
270.78 \\
270.54 \\
270.83\end{array}$ & $\begin{array}{l}403.61 * \\
403.61 \\
403.85 \\
403.56\end{array}$ \\
\hline $699-43-43$ & $\begin{array}{l}4 / 18 / 91 \\
5 / 23 / 91 \\
6 / 13 / 91\end{array}$ & $\begin{array}{l}164.34 \\
164.21 \\
164.20\end{array}$ & $\begin{array}{l}415.03 \\
415.16 \\
415.17\end{array}$ \\
\hline $699-43-45$ & $\begin{array}{l}4 / 18 / 91 \\
5 / 23 / 91 \\
6 / 13 / 91\end{array}$ & $\begin{array}{l}192.93 \\
193.03 \\
192.91\end{array}$ & $\begin{array}{l}404.75 \\
404.65 \\
404.77\end{array}$ \\
\hline \multicolumn{4}{|c|}{ Supplemental Wells for Assessment Monitoring } \\
\hline $299-E 25-10$ & $6 / 10 / 91$ & 252.24 & 403.60 \\
\hline $299-E 25-11$ & $6 / 10 / 91$ & 277.79 & 403.49 \\
\hline $299-E 25-18$ & $6 / 10 / 91$ & 275.50 & 403.55 \\
\hline $299-E 25-19$ & $6 / 10 / 91$ & 273.78 & 403.42 \\
\hline $299-E 25-20$ & $6 / 10 / 91$ & 272.91 & 403.39 \\
\hline
\end{tabular}


Table 6-1. RCRA Water Level Measurement Report 216-A-29 Ditch - Second Quarter 1991. (sheet 2 of 2)

\begin{tabular}{cccc}
\hline Wel1 & Date & $\begin{array}{c}\text { Depth to water } \\
(\mathrm{ft})\end{array}$ & $\begin{array}{c}\text { Water Tevel elevation } \\
\text { above ms }(\mathrm{ft})\end{array}$ \\
\hline $299-$ E25-31 & $6 / 10 / 91$ & 268.85 & 403.68 \\
& $6 / 25 / 91$ & 268.78 & $403.75^{\star}$ \\
$299-$ E25-36 & $6 / 20 / 91$ & 304.15 & 403.24 \\
$299-$ E25-6 & $6 / 10 / 91$ & 257.32 & 400.99 \\
$299-E 25-9$ & $6 / 10 / 91$ & 253.31 & 401.55 \\
$299-E 26-2$ & $6 / 10 / 91$ & 231.36 & 403.94 \\
\hline
\end{tabular}

NOTES: 1. Water level elevations are calculated by subtracting the measured depth to water from the surveyed elevation for the well.

2. Depth-to-water values are transcribed from field records.

3. Elevations marked with an $1 * 1$ were measured at the time of sampling. 
DOE/RL-91-47

This page intentionally left blank. 
DOE/RL-91-47

\subsection{6-A-10 Crib}

D. K. Tyler

Westinghouse Hanford Company

The groundwater monitoring network for the 216-A-10 Crib has not changed from that described in the quarterly report for the period July 1 through September 30, 1990 (DOE-RL 1990). Please reference that quarterly for a description of the monitoring network.

Water level measurements for the second quarter of 1991 are reported in Table 7-1. 
$\mathrm{DOE} / \mathrm{RL}-91-47$

Table 7-1. RCRA Water Level Measurement Report 216-A-10 Crib Second Quarter 1991.

\begin{tabular}{lccc}
\hline We11 & Date & $\begin{array}{c}\text { Depth to water } \\
(\mathrm{ft})\end{array}$ & $\begin{array}{c}\text { Water level elevation } \\
\text { above ms }(\mathrm{ft})\end{array}$ \\
\hline $299-E 17-1$ & $6 / 20 / 91$ & 316.27 & 402.90 \\
$299-E 17-19$ & $6 / 20 / 91$ & 316.69 & 402.64 \\
$299-E 17-20$ & $6 / 20 / 91$ & 316.40 & 402.83 \\
$299-E 24-16$ & $6 / 20 / 91$ & 315.19 & 403.08 \\
$299-E 24-17$ & $6 / 20 / 91$ & 316.53 & 402.16 \\
$299-E 24-18$ & $6 / 20 / 91$ & 316.08 & 403.20 \\
$299-E 24-2$ & $6 / 20 / 91$ & 314.54 & 402.93 \\
$299-E 25-36$ & $6 / 20 / 91$ & 304.15 & 403.24 \\
\hline
\end{tabular}

NOTES: 1. Water level elevations are calculated by subtracting the measured depth to water from the surveyed elevation for the well.

2. Depth-to-water values are transcribed from field records.

3. Elevations marked with an $1 * 1$ were measured at the time of sampling. 
DOE/RL-91-47

\subsection{6-A-36B Crib}

D. K. Tyler

Westinghouse Hanford Company

The groundwater monitoring network for the 216-A-36B Crib has not changed from that described in the quarterly report for the period July 1 through September 30, 1990 (DOE-RL 1990). Please reference that quarterly for a description of the monitoring network.

Water level measurements are the only activities that have occurred during the second quarter of 1991. These data are reported in Table 8-1. 


$$
\mathrm{DOE} / \mathrm{RL}-91-47
$$

Table 8-1. RCRA Watel Level Measurement Report 216-A-36B Crib Second Quarter 1991.

\begin{tabular}{cccc}
\hline Wel1 & Date & $\begin{array}{c}\text { Depth of water } \\
(\mathrm{ft})\end{array}$ & $\begin{array}{c}\text { Water level elevation } \\
\text { above ms }(\mathrm{ft})\end{array}$ \\
\hline $299-E 17-14$ & $6 / 20 / 91$ & 319.06 & 403.12 \\
$299-E 17-15$ & $6 / 20 / 91$ & 319.35 & 402.43 \\
$299-E 17-16$ & $6 / 20 / 91$ & 317.58 & 403.00 \\
$299-E 17-17$ & $6 / 20 / 91$ & 316.83 & $403 . n 9$ \\
$299-E 1: 18$ & $6 / 20 / 91$ & 317.41 & 403.24 \\
$299-E 17-5$ & $6 / 20 / 91$ & 316.02 & 402.67 \\
$299-E 17-6$ & $6 / 20 / 91$ & 314.66 & 402.98 \\
\hline
\end{tabular}

NOTES: 1. Water lev's elevations are calculated by subtracting the measured depth to water from the surveyed elevation for the well.

2. Depth-to-water values are transcribed from field racords.

3. Elevations marked with an $1 * 1$ were measured at the time of sampling. 
$\mathrm{DOE} / \mathrm{RL}-91-47$

\subsection{6-B-3 POND SYSTEM}

C. D. Delaney

Westinghouse Hanford Company

There have been no changes to the 216-B-3 Pond System groundwater monitoring network from that described in the quarterly report for the period July 1 through September 30, 1990 (DOE-RL 1990). Only one well from the 216-B-3 Pond System groundwater monitoring network was sampled between April 1 and June 30, 1991. We11 699-43-41E was sampled on June 28, 1991 and will be discussed in the next quarterly report.

Water level data collected for the second quarter of 1991 are presented in Table 9-1. 
Table 9-1. RCRA Water Level Measurement Report 216-B-3 Pond System - Second Quarter 1991. (sheet 1 of 2)

\begin{tabular}{|c|c|c|c|}
\hline We11 & Date & $\begin{array}{l}\text { Depth water } \\
(\mathrm{ft})\end{array}$ & $\begin{array}{c}\text { Water level elevation } \\
\text { above msl (ft) }\end{array}$ \\
\hline \multicolumn{4}{|c|}{ Wells Monitoring the Unconfined Aquifer at the Water Table } \\
\hline 299-E18-1 & $6 / 05 / 91$ & 316.74 & 403.50 \\
\hline 299-E32-4 & $\begin{array}{l}4 / 19 / 91 \\
5 / 24 / 91 \\
6 / 12 / 91\end{array}$ & $\begin{array}{l}282.84 \\
282.83 \\
282.83\end{array}$ & $\begin{array}{l}403.04 \\
403.05 \\
403.05\end{array}$ \\
\hline $699-42-40 A$ & $\begin{array}{l}4 / 18 / 91 \\
5 / 23 / 91 \\
6 / 13 / 91\end{array}$ & $\begin{array}{l}123.04 \\
122.59 \\
122.84\end{array}$ & $\begin{array}{l}422.49 \\
422.94 \\
422.69\end{array}$ \\
\hline $699-43-42 \mathrm{~J}$ & $\begin{array}{l}4 / 18 / 91 \\
5 / 23 / 91 \\
6 / 13 / 91\end{array}$ & $\begin{array}{l}162.48 \\
162.14 \\
162.37\end{array}$ & $\begin{array}{l}419.20 \\
419.54 \\
419.31\end{array}$ \\
\hline $699-43-43$ & $\begin{array}{l}4 / 18 / 91 \\
5 / 23 / 91 \\
6 / 13 / 91\end{array}$ & $\begin{array}{l}164.34 \\
164.21 \\
164.20\end{array}$ & $\begin{array}{l}415.03 \\
415.16 \\
415.17\end{array}$ \\
\hline $699-43-45$ & $\begin{array}{l}4 / 18 / 91 \\
5 / 23 / 91 \\
6 / 13 / 91\end{array}$ & $\begin{array}{l}192.93 \\
193.03 \\
192.91\end{array}$ & $\begin{array}{l}404.75 \\
404.65 \\
404.77\end{array}$ \\
\hline $699-44-42$ & $\begin{array}{l}4 / 18 / 91 \\
5 / 23 / 91 \\
6 / 13 / 91\end{array}$ & $\begin{array}{l}158.33 \\
158.24 \\
158.29\end{array}$ & $\begin{array}{l}420.89 \\
420.98 \\
420.93\end{array}$ \\
\hline $699-44-43 B$ & $\begin{array}{l}4 / 18 / 91 \\
5 / 23 / 91 \\
6 / 13 / 91\end{array}$ & $\begin{array}{l}164.65 \\
164.62 \\
164.48\end{array}$ & $\begin{array}{l}415.47 \\
415.50 \\
415.64\end{array}$ \\
\hline \multicolumn{4}{|c|}{ Wells Monitoring the Confined or Semi-Confined Aquifer } \\
\hline $699-40-39$ & $\begin{array}{l}4 / 15 / 91 \\
4 / 18 / 91 \\
5 / 23 / 91 \\
6 / 13 / 91\end{array}$ & $\begin{array}{l}128.21 \\
128.31 \\
128.42 \\
128.39\end{array}$ & $\begin{array}{l}413.57^{\star} \\
413.53 \\
413.42 \\
413.45\end{array}$ \\
\hline $699-41-40$ & $\begin{array}{l}4 / 15 / 91 \\
4 / 18 / 91 \\
5 / 23 / 91 \\
6 / 13 / 91\end{array}$ & $\begin{array}{l}129.68 \\
129.78 \\
129.80 \\
129.56\end{array}$ & $\begin{array}{l}416.26^{\star} \\
416.16 \\
416.14 \\
416.38\end{array}$ \\
\hline $699-43-41 E$ & $\begin{array}{l}4 / 18 / 91 \\
4 / 18 / 91 \\
5 / 23 / 91 \\
6 / 13 / 91 \\
6 / 28 / 91\end{array}$ & $\begin{array}{l}129.76 \\
129.71 \\
129.51 \\
129.38 \\
129.22\end{array}$ & $\begin{array}{l}421.10^{\star} \\
421.15 \\
421.35 \\
421.48 \\
421.64^{\star}\end{array}$ \\
\hline
\end{tabular}




$$
\mathrm{DOE} / \mathrm{RL}-91-47
$$

Table 9-1. RCRA Water Level Measurement Report 216-B-3 Pond System - Second Quarter 1991. (sheet 2 of 2)

\begin{tabular}{|c|c|c|c|}
\hline Well & Date & $\begin{array}{c}\text { Depth water } \\
(\mathrm{ft})\end{array}$ & $\begin{array}{c}\text { Water level elevation } \\
\text { above msl (ft) }\end{array}$ \\
\hline $699-43-41 F$ & $\begin{array}{l}4 / 18 / 91 \\
4 / 18 / 91 \\
5 / 23 / 91 \\
6 / 13 / 91\end{array}$ & $\begin{array}{l}129.75 \\
129.72 \\
129.33 \\
129.47\end{array}$ & $\begin{array}{l}421.26^{\star} \\
421.29 \\
421.68 \\
421.54\end{array}$ \\
\hline \multicolumn{4}{|c|}{ Well Monitoring the Unconfined Aquifer Below the Water Table } \\
\hline $699-42-42 B$ & $\begin{array}{l}4 / 18 / 91 \\
5 / 23 / 91 \\
6 / 13 / 91\end{array}$ & $\begin{array}{l}166.03 \\
165.82 \\
165.83\end{array}$ & $\begin{array}{l}417.20 \\
417.41 \\
417.40\end{array}$ \\
\hline \multicolumn{4}{|c|}{$\begin{array}{l}\text { NOTES: 1. Water level elevations are calculated by subtracting the } \\
\text { measured depth to water from the surveyed elevation for } \\
\text { the well. } \\
\text { 2. Depth-to-water values are transcribed from field records. } \\
\text { 3. Elevations marked with an } * * \text { were measured at the time of } \\
\text { sampling. }\end{array}$} \\
\hline
\end{tabular}


DOE/RL-91-47

This page intentionally left blank. 


\subsection{1-M POND}

\section{A. Chamness \\ Pacific Northwest Laboratory}

The groundwater monitoring network for the 2101-M Pond has not changed from that described in the quarterly report for the period July 1 through September 30, 1990 (DOE-RL 1990). Please reference that quarterly for a description of the monitoring network.

Water level data collected for the second quarter of 1991 are presented in Table 10-1. 
Table 10-1. RCRA Water Level Measurement Report 2101-M Pond - Second Quarter 1991.

\begin{tabular}{cccc}
\hline We11 & Date & $\begin{array}{c}\text { Depth to water } \\
(\mathrm{ft})\end{array}$ & $\begin{array}{c}\text { Water level elevation } \\
\text { above ms } 1 \text { (ft) }\end{array}$ \\
\hline $299-$ E18-1 & $6 / 05 / 91$ & 316.74 & 403.50 \\
$299-E 18-3$ & $6 / 05 / 91$ & 318.58 & 403.46 \\
\hline
\end{tabular}

NOTES: 1. Water level elevations are calculated by subtracting the measured depth to water from the surveyed elevation for the well.

2. Depth-to-water values are transcribed from field records. 


\subsection{GROUT TREATMENT FACILITY \\ D. K. Tyler \\ Westinghouse Hanford Company}

The groundwater monitoring network for the Grout Treatment Facility has not changed from that described in the quarterly report for the period October 1, 1990 through December 31, 1990 (DOE-RL 1991a). Please reference that quarterly for a description of the monitoring network. During the second quarter of 1991, water levels were measured and water chemistry samples were
collected.

Water chemistry samples were collected in the last days of the second quarter. Analytical results were not available for verification at the time this report was prepared; these will be included in the next quarterly report.

Water level measurements obtained for the second quarter of 1991 are listed in Table 11-1. Some water level data for wells 299-E25-28, 299-E25-29P, and 299-E25-33 are suspected of being in error. A review is being performed to determine the source of error and to make corrections, if appropriate. 
Table 11-1. RCRA Water Level Measurement Report Grout Treatment Facility - Second Quarter 1991. (sheet 1 of 2)

\begin{tabular}{|c|c|c|c|}
\hline Well & Date & $\begin{array}{c}\text { Depth of water } \\
(\mathrm{ft})\end{array}$ & $\begin{array}{l}\text { Water level elevation } \\
\text { above msl (ft) }\end{array}$ \\
\hline 299-E25-18 & $6 / 10 / 91$ & 275.50 & 403.55 \\
\hline 299-E25-20 & $6 / 10 / 91$ & 272.91 & 403.39 \\
\hline 299-E25-24 & $6 / 25 / 91$ & 275.66 & 403.89 \\
\hline 299-E25-25 & $\begin{array}{l}6 / 25 / 91 \\
6 / 25 / 91\end{array}$ & $\begin{array}{l}264.96 \\
264.93\end{array}$ & $\begin{array}{l}404.46 \\
404.49 *\end{array}$ \\
\hline $299-E 25-26$ & $\begin{array}{l}4 / 17 / 91 \\
4 / 18 / 91 \\
5 / 24 / 91 \\
6 / 13 / 91\end{array}$ & $\begin{array}{l}264.60 \\
264.54 \\
264.62 \\
264.58\end{array}$ & $\begin{array}{l}403.92^{\star} \\
403.98 \\
403.90 \\
403.94\end{array}$ \\
\hline $299-E 25-27$ & $6 / 25 / 91$ & 272.11 & 403.97 \\
\hline 299-E25-28 & $\begin{array}{l}4 / 10 / 91 \\
4 / 17 / 91 \\
4 / 18 / 91 \\
5 / 24 / 91 \\
6 / 13 / 91\end{array}$ & $\begin{array}{l}258.62 \\
258.25 \\
258.37 \\
258.38 \\
252.44\end{array}$ & $\begin{array}{l}403.82^{\star} \\
404.19^{\star} \\
404.07 \\
404.06 \\
410.00-x\end{array}$ \\
\hline 299-E25-29P & $\begin{array}{l}6 / 25 / 91 \\
6 / 25 / 91\end{array}$ & $\begin{array}{l}271.58 \\
269.76\end{array}$ & $\begin{array}{l}401.28-x \\
403.10 *\end{array}$ \\
\hline 299-E25-29Q & $6 / 25 / 91$ & 268.95 & 403.67 \\
\hline 299-E25-30Q & $6 / 25 / 91$ & 274.44 & 403.47 \\
\hline $299-E 25-31$ & $\begin{array}{l}6 / 10 / 91 \\
6 / 25 / 91\end{array}$ & $\begin{array}{l}268.85 \\
268.78\end{array}$ & $\begin{array}{l}403.68 \\
403.75^{\star}\end{array}$ \\
\hline $299-E 25-32 P$ & $\begin{array}{l}4 / 11 / 91 \\
4 / 18 / 91 \\
5 / 24 / 91 \\
6 / 13 / 91\end{array}$ & $\begin{array}{l}266.15 \\
266.17 \\
266.04 \\
266.12\end{array}$ & $\begin{array}{l}403.89 * \\
403.87 \\
404.00 \\
403.92\end{array}$ \\
\hline 299-E25-32Q & $6 / 25 / 91$ & 265.98 & 404.06 \\
\hline 299-E25-33 & $\begin{array}{l}6 / 25 / 91 \\
6 / 27 / 91\end{array}$ & $\begin{array}{l}246.42 \\
256.29\end{array}$ & $\begin{array}{l}403.61 \\
393.74^{\star}-x\end{array}$ \\
\hline 299-E25-37 & $\begin{array}{l}6 / 25 / 91 \\
6 / 26 / 91\end{array}$ & $\begin{array}{l}269.67 \\
269.68\end{array}$ & $\begin{array}{l}403.62 \\
403.61^{\star}\end{array}$ \\
\hline
\end{tabular}


Table 11-1. RCRA Water Level Measurement Report Grout Treatment Facility - Second Quarter 1991. (sheet 2 of 2)

\begin{tabular}{cccc}
\hline We11 & Date & $\begin{array}{c}\text { Depth of water } \\
(\mathrm{ft})\end{array}$ & $\begin{array}{c}\text { Water level elevation } \\
\text { above ms } 1 \text { (ft) }\end{array}$ \\
\hline $299-$ E25-38 & $6 / 25 / 91$ & 269.70 & 403.82 \\
& $6 / 26 / 91$ & 269.65 & $403.87 *$ \\
$299-E 25-39$ & $6 / 10 / 91$ & 266.58 & 404.65 \\
& $6 / 26 / 91$ & 266.86 & $404.37 *$ \\
\hline
\end{tabular}

NOTES: 1. Water level elevations are calculated by subtracting the measured depth to water from the surveyed elevation for the wel1.

2. Depth-to-water values are transcribed from field records.

3. Elevations marked with an ' $*$ ' were measured at the time of sampling.

4. Data suspected of being in error are marked ' $-X$ ' and are under review. 
DOE/RL-91-47

This page intentionally left blank. 


\subsection{SINGLE-SHELL TANKS}

J. A. Caggiano

Westinghouse Hanford Company

The groundwater monitoring network for the Single-Shell Tanks (SST) is being expanded with the addition of 10 new RCRA Standard wells at Waste Management Areas B-BX-BY, S-SX, TX-TY, and $U$. Construction of these 10 new RCRA groundwater monitoring wells is scheduled to begin in August 1991. Water levels measured in 30 wells for the quarter are provided in Table 12-1.

The last of the eleven calendar year 1990 RCRA standard groundwater monitoring wells were completed at the SSTs in June 1991. No other field activities took place this quarter. Wells 299-E33-38 and 299-E33-39, CERCLA remedial investigation wells for the 200-BP-1 Operable Unit, have been completed in the unconfined aquifer on the northern margin of Waste Management Area (WMA) B-BX-BY. Well 299-E33-36, a downgradient RCRA standard we 11 for the 216-B-63 Trench, also will be used for groundwater monitoring. Water level and some constituent data will be obtained from these wells as part of the SST network for WMA B-BX-BY. There are now 26 RCRA standard groundwater monitoring wells at the SSTs (including wells 299-E33-36, 299-E33-38, and 299-E33-39). 
Table 12-1. RCRA Water Level Measurement Report Single-Shell Tanks - Second Quarter 1991. (sheet 1 of 3)

\begin{tabular}{|c|c|c|c|}
\hline We11 & Date & $\begin{array}{c}\text { Depth to water } \\
(\mathrm{ft})\end{array}$ & $\begin{array}{c}\text { Water level elevation } \\
\text { above msl (ft) }\end{array}$ \\
\hline \multicolumn{4}{|c|}{ 241-A/AX Tank Farm Wells } \\
\hline 299-E24-13 & $\begin{array}{l}4 / 25 / 91 \\
5 / 22 / 91 \\
6 / 17 / 91\end{array}$ & $\begin{array}{l}287.66 \\
287.63 \\
287.82\end{array}$ & $\begin{array}{l}403.47 \\
403.50 \\
403.31\end{array}$ \\
\hline 299-E24-19 & $\begin{array}{l}4 / 24 / 91 \\
5 / 21 / 91 \\
6 / 17 / 91\end{array}$ & $\begin{array}{l}290.27 \\
290.28 \\
290.25\end{array}$ & $\begin{array}{l}403.38 \\
403.37 \\
403.40\end{array}$ \\
\hline 299-E25-1 & $\begin{array}{l}4 / 25 / 91 \\
5 / 22 / 91 \\
6 / 17 / 91\end{array}$ & $\begin{array}{l}287.04 \\
287.04 \\
287.25\end{array}$ & $\begin{array}{l}403.53 \\
403.53 \\
403.32\end{array}$ \\
\hline $299-E 25-13$ & $\begin{array}{l}4 / 25 / 91 \\
5 / 22 / 91 \\
6 / 04 / 91 \\
6 / 17 / 91\end{array}$ & $\begin{array}{l}278.90 \\
278.89 \\
278.73 \\
279.10\end{array}$ & $\begin{array}{l}403.53 \\
403.54 \\
403.70 * \\
403.33\end{array}$ \\
\hline $299-E 25-15$ & $\begin{array}{l}4 / 25 / 91 \\
5 / 22 / 91 \\
6 / 17 / 91\end{array}$ & $\begin{array}{l}286.21 \\
286.21 \\
286.40\end{array}$ & $\begin{array}{l}403.52 \\
403.52 \\
403.33\end{array}$ \\
\hline $299-E 25-2$ & $\begin{array}{l}4 / 24 / 91 \\
5 / 21 / 91 \\
6 / 19 / 91\end{array}$ & $\begin{array}{l}272.04 \\
271.84 \\
272.11\end{array}$ & $\begin{array}{l}403.41 \\
403.61 \\
403.34\end{array}$ \\
\hline $299-E 25-40$ & $\begin{array}{l}4 / 24 / 91 \\
5 / 20 / 91 \\
6 / 17 / 91\end{array}$ & $\begin{array}{l}262.16 \\
262.16 \\
262.22\end{array}$ & $\begin{array}{l}403.55 \\
403.55 \\
403.49\end{array}$ \\
\hline $299-E 25-41$ & $\begin{array}{l}4 / 24 / 91 \\
5 / 20 / 91 \\
6 / 17 / 91\end{array}$ & $\begin{array}{l}267.64 \\
267.66 \\
267.74\end{array}$ & $\begin{array}{l}403.62 \\
403.60 \\
403.52\end{array}$ \\
\hline \multicolumn{4}{|c|}{ 241-B/BX/BY Tank Farm Wells } \\
\hline $299-E 33-18$ & $\begin{array}{l}4 / 24 / 91 \\
5 / 21 / 91 \\
6 / 19 / 91\end{array}$ & $\begin{array}{l}240.37 \\
240.25 \\
240.30\end{array}$ & $\begin{array}{l}403.25 \\
403.37 \\
403.32\end{array}$ \\
\hline $299-E 33-21$ & $\begin{array}{l}4 / 24 / 91 \\
5 / 20 / 91 \\
6 / 17 / 91\end{array}$ & $\begin{array}{l}264.88 \\
264.86 \\
264.86\end{array}$ & $\begin{array}{l}403.25 \\
403.27 \\
403.27\end{array}$ \\
\hline
\end{tabular}


Table 12-1. RCRA Water Level Measurement Report Single-Shell Tanks - Second Quarter 1991. (sheet 2 of 3 )

\begin{tabular}{|c|c|c|c|}
\hline Well & Date & $\begin{array}{c}\text { Depth to water } \\
(\mathrm{ft})\end{array}$ & $\begin{array}{c}\text { Water level elevation } \\
\text { above } \mathrm{msl}(\mathrm{ft})\end{array}$ \\
\hline 299-E33-24 & $\begin{array}{l}4 / 24 / 91 \\
5 / 21 / 91 \\
6 / 19 / 91\end{array}$ & $\begin{array}{l}234.35 \\
234.38 \\
234.43\end{array}$ & $\begin{array}{l}403.35 \\
403.32 \\
403.27\end{array}$ \\
\hline 299-E33-31 & $\begin{array}{l}4 / 25 / 91 \\
5 / 20 / 91 \\
6 / 17 / 91\end{array}$ & $\begin{array}{l}244.02 \\
244.04 \\
244.03\end{array}$ & $\begin{array}{l}403.26 \\
403.24 \\
403.25\end{array}$ \\
\hline 299-E33-32 & $\begin{array}{l}4 / 24 / 91 \\
5 / 20 / 91 \\
6 / 17 / 91\end{array}$ & $\begin{array}{l}256.72 \\
256.68 \\
256.67\end{array}$ & $\begin{array}{l}403.11 \\
403.15 \\
403.16\end{array}$ \\
\hline 299-E33-33 & $\begin{array}{l}4 / 24 / 91 \\
5 / 20 / 91 \\
6 / 17 / 91\end{array}$ & $\begin{array}{l}236.87 \\
236.94 \\
236.80\end{array}$ & $\begin{array}{l}403.30 \\
403.23 \\
403.37\end{array}$ \\
\hline 299-E33-8 & $\begin{array}{l}4 / 25 / 91 \\
5 / 20 / 91 \\
6 / 17 / 91\end{array}$ & $\begin{array}{l}247.45 \\
247.45 \\
247.48\end{array}$ & $\begin{array}{l}403.28 \\
403.28 \\
403.25\end{array}$ \\
\hline \multicolumn{4}{|c|}{ 241-C Tank Farm Wells } \\
\hline 299-E27-12 & $\begin{array}{l}4 / 24 / 91 \\
5 / 20 / 91 \\
6 / 17 / 91\end{array}$ & $\begin{array}{l}257.57 \\
257.58 \\
257.61\end{array}$ & $\begin{array}{l}403.38 \\
403.37 \\
403.34\end{array}$ \\
\hline 299-E27-13 & $\begin{array}{l}4 / 24 / 91 \\
5 / 20 / 91 \\
6 / 17 / 91\end{array}$ & $\begin{array}{l}265.68 \\
265.73 \\
265.74\end{array}$ & $\begin{array}{l}403.31 \\
403.26 \\
403.25\end{array}$ \\
\hline 299-E27-14 & $\begin{array}{l}4 / 24 / 91 \\
5 / 20 / 91 \\
6 / 17 / 91\end{array}$ & $\begin{array}{l}254.78 \\
254.89 \\
254.82\end{array}$ & $\begin{array}{l}403.56 \\
403.45 \\
403.52\end{array}$ \\
\hline 299-E27-15 & $\begin{array}{l}4 / 24 / 91 \\
5 / 20 / 91 \\
6 / 17 / 91\end{array}$ & $\begin{array}{l}249.59 \\
249.65 \\
249.67\end{array}$ & $\begin{array}{l}403.08 \\
403.02 \\
403.00\end{array}$ \\
\hline $299-E 27-7$ & $\begin{array}{l}4 / 24 / 91 \\
5 / 20 / 91 \\
6 / 17 / 91\end{array}$ & $\begin{array}{l}231.13 \\
231.12 \\
231.15\end{array}$ & $\begin{array}{l}403.54 \\
403.55 \\
403.52\end{array}$ \\
\hline
\end{tabular}


DOE/RL-91-47

Table 12-1. RCRA Water Level Measurement Report Single-Shell Tanks - Second Quarter 1991. (sheet 3 of 3 )

\begin{tabular}{|c|c|c|c|}
\hline Well & Date & $\begin{array}{c}\text { Depth to water } \\
(\mathrm{ft})\end{array}$ & $\begin{array}{l}\text { Water level elevation } \\
\text { above msl (ft) }\end{array}$ \\
\hline \multicolumn{4}{|c|}{ 241-T Tank Farm Wells } \\
\hline $299-W 10-10$ & $\begin{array}{l}4 / 24 / 91 \\
5 / 21 / 91 \\
6 / 17 / 91\end{array}$ & $\begin{array}{l}210.97 \\
211.02 \\
211.38\end{array}$ & $\begin{array}{l}464.12 \\
464.07 \\
463.71\end{array}$ \\
\hline $299-W 10-11$ & $\begin{array}{l}4 / 24 / 91 \\
5 / 21 / 91 \\
6 / 17 / 91\end{array}$ & $\begin{array}{l}210.87 \\
210.88 \\
211.26\end{array}$ & $\begin{array}{l}464.39 \\
464.38 \\
464.00\end{array}$ \\
\hline $2 \subseteq 9-W 10-12$ & $\begin{array}{l}4 / 24 / 91 \\
5 / 21 / 91 \\
6 / 17 / 91\end{array}$ & $\begin{array}{l}210.58 \\
210.70 \\
210.98\end{array}$ & $\begin{array}{l}464.17 \\
464.05 \\
463.77\end{array}$ \\
\hline 299-W10-15 & $\begin{array}{l}4 / 24 / 91 \\
5 / 21 / 91 \\
6 / 17 / 91\end{array}$ & $\begin{array}{l}211.53 \\
211.55 \\
211.92\end{array}$ & $\begin{array}{l}464.11 \\
464.09 \\
463.72\end{array}$ \\
\hline 299-W10-1's & $\begin{array}{l}4 / 24 / 91 \\
5 / 21 / 91 \\
6 / 17 / 91\end{array}$ & $\begin{array}{l}208.34 \\
208.36 \\
208.65\end{array}$ & $\begin{array}{l}464.42 \\
464.40 \\
464.11\end{array}$ \\
\hline $299-W 10-3$ & $\begin{array}{l}4 / 24 / 91 \\
5 / 21 / 91 \\
6 / 18 / 91\end{array}$ & $\begin{array}{l}207.01 \\
208.17 \\
207.32\end{array}$ & $\begin{array}{l}464.45 \\
463.29-x \\
464.14\end{array}$ \\
\hline $299-W 10-8$ & $\begin{array}{l}4 / 24 / 91 \\
5 / 21 / 91 \\
6 / 19 / 91\end{array}$ & $\begin{array}{l}216.24 \\
216.29 \\
216.34\end{array}$ & $\begin{array}{l}463.81 \\
463.76 \\
463.71\end{array}$ \\
\hline 299-W10-9 & $\begin{array}{l}4 / 24 / 91 \\
5 / 21 / 91 \\
6 / 17 / 91\end{array}$ & $\begin{array}{l}210.52 \\
213.95 \\
210.83\end{array}$ & $\begin{array}{l}464.15 \\
460.72 \\
463.84\end{array}$ \\
\hline $299-W 11-23$ & $\begin{array}{l}4 / 24 / 91 \\
5 / 21 / 91 \\
6 / 19 / 91\end{array}$ & $\begin{array}{l}222.03 \\
222.05 \\
222.06\end{array}$ & $\begin{array}{l}463.39 \\
463.37 \\
463.36\end{array}$ \\
\hline $299-W 11-24$ & $\begin{array}{l}4 / 24 / 91 \\
5 / 21 / 91 \\
6 / 19 / 91\end{array}$ & $\begin{array}{l}223.30 \\
223.35 \\
223.34\end{array}$ & $\begin{array}{l}463.87 \\
463.82 \\
463.83\end{array}$ \\
\hline
\end{tabular}

NOTES: 1. Water level elevations are calculated by subtracting the measured depth to water from the surveyed elevation for the well.

2. Depth-to-water values are transcribed from field records.

3. Elevations marked with an $\mid * 1$ were measured at the time of sampling.

4. Data suspected of being in error are marked ' $-X$ ' and are under review. 
DOE/RL-91-47

\subsection{NONRADIOACTIVE DANGEROUS WASTE LANDFILL}

F. N. Hodges

Westinghouse Hanford Company

The groundwater monitoring network for the Nonradioactive Dangerous Waste Landfill (NRDWL) has not changed from that described in the quarterly report for the period July 1 through September 30, 1991 (DOE-RL 1990). Please reference that quarterly for a description of the monitoring network. No monitoring activities other than water level measurements have taken place during the second quarter of 1991.

The water level data collected for the second quarter of 1991 are presented in Table 13-1. 
Table 13-1. RCRA Water Level Measurement Report NRDWL - Second Quarter 1991. (sheet 1 of 2)

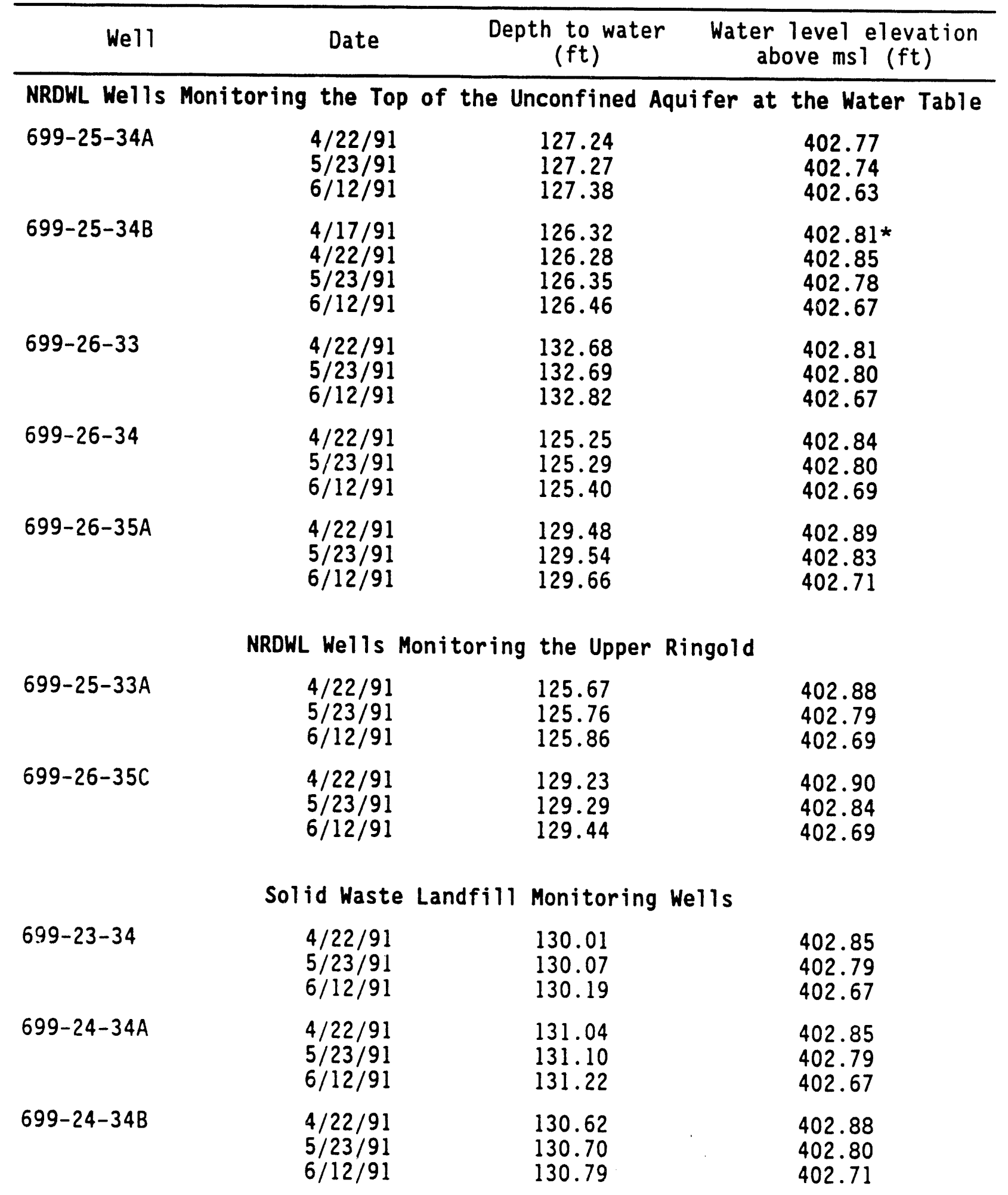


$\mathrm{DOE} / \mathrm{RL}-91-47$

Table 13-1. RCRA Water Level Measurement Report NRDWL - Second Quarter 1991. (sheet 2 of 2)

\begin{tabular}{|c|c|c|c|}
\hline Well & Date & $\begin{array}{c}\text { Depth to water } \\
(\mathrm{ft})\end{array}$ & $\begin{array}{c}\text { Water level elevation } \\
\text { above msl (ft) }\end{array}$ \\
\hline $699-24-34 C$ & $\begin{array}{l}4 / 22 / 91 \\
5 / 23 / 91 \\
6 / 12 / 91\end{array}$ & $\begin{array}{l}129.76 \\
129.81 \\
129.92\end{array}$ & $\begin{array}{l}402.82 \\
402.77 \\
402.66\end{array}$ \\
\hline $699-24-35$ & $\begin{array}{l}4 / 22 / 91 \\
5 / 23 / 91 \\
6 / 12 / 91\end{array}$ & $\begin{array}{l}135.94 \\
136.00 \\
136.10\end{array}$ & $\begin{array}{l}402.87 \\
402.81 \\
402.71\end{array}$ \\
\hline $699-25-34 C$ & $\begin{array}{l}4 / 22 / 91 \\
5 / 23 / 91 \\
6 / 12 / 91\end{array}$ & $\begin{array}{l}132.61 \\
132.67 \\
132.77\end{array}$ & $\begin{array}{l}402.85 \\
402.79 \\
402.69\end{array}$ \\
\hline \multicolumn{4}{|c|}{ Other Wells Associated with NRDWL } \\
\hline $699-20-20$ & $\begin{array}{l}4 / 22 / 91 \\
5 / 23 / 91 \\
6 / 12 / 91\end{array}$ & $\begin{array}{l}104.28 \\
104.34 \\
104.48\end{array}$ & $\begin{array}{l}401.30 \\
401.24 \\
401.10\end{array}$ \\
\hline $699-20-39$ & $\begin{array}{l}4 / 22 / 91 \\
5 / 23 / 91 \\
6 / 12 / 91\end{array}$ & $\begin{array}{l}136.74 \\
136.87 \\
136.96\end{array}$ & $\begin{array}{l}403.24 \\
403.11 \\
403.02\end{array}$ \\
\hline $699-24-33$ & $\begin{array}{l}4 / 22 / 91 \\
5 / 23 / 91 \\
6 / 12 / 91 \\
6 / 20 / 91\end{array}$ & $\begin{array}{l}121.43 \\
121.50 \\
121.63 \\
121.47\end{array}$ & $\begin{array}{l}402.78 \\
402.71 \\
402.58 \\
402.74 \star\end{array}$ \\
\hline $699-28-40$ & $\begin{array}{l}4 / 22 / 91 \\
5 / 23 / 91 \\
6 / 12 / 91\end{array}$ & $\begin{array}{l}156.17 \\
156.30 \\
156.40\end{array}$ & $\begin{array}{l}403.27 \\
403.14 \\
403.04\end{array}$ \\
\hline $699-31-31$ & $\begin{array}{l}4 / 22 / 91 \\
5 / 23 / 91 \\
6 / 12 / 91\end{array}$ & $\begin{array}{l}126.55 \\
126.62 \\
126.72\end{array}$ & $\begin{array}{l}402.77 \\
402.70 \\
402.60\end{array}$ \\
\hline $699-34-39 A$ & $\begin{array}{l}4 / 22 / 91 \\
5 / 23 / 91 \\
6 / 12 / 91\end{array}$ & $\begin{array}{l}133.75 \\
133.85 \\
133.93\end{array}$ & $\begin{array}{l}403.32 \\
403.22 \\
403.14\end{array}$ \\
\hline
\end{tabular}

NOTES: 1. Water level elevations are calculated by subtracting the measured depth to water from the surveyed elevation for the well.

2. Depth-to-water values are transcribed from field records.

3. Elevations marked with an ' $*$ ' were measured at the time of sampling. 
DOE/RL-91-47

This page intentionally left blank. 


\subsection{REFERENCES}

Comprehensive Environmental Response, Compensation and Liability Act of 1980, Public Law 96-510, 94 Stat. 2767, 42 USC 9601 et seq.

DOE-RL, 1990, Quarterly Report of RCRA Groundwater Monitoring Data for Period July 1, 1990 through September 30, 1990, DOE/RL-90-46, U.S. Department of Energy-Richland Operations Office, Richland, Washington.

DOE-RL, 199la, Quarterly Report of RCRA Groundwater Monitoring Data for Period October 1, 1990 through December 31, 1990, DOE/RL-91-04, U.S. Department of Energy-Richland Operations Office, Richland, Washington.

DOE-RL, 1991b, Quarterly Report of RCRA Groundwater Monitoring Data for Period January 1, 1991 through March 31, 1991, D0E/RL-91-26, U.S. Department of Energy-Richland Operations Office, Richland, Washington.

Ecology, 1991, Dangerous Waste Regulations, Washington Administrative Code WAC 173-303, Washington State Department of Ecology, 0lympia, Washington.

EPA, 1989, Interim Status Standards for Owners and Operators of Hazardous Waste Treatment, Storage, and Disposal Facilities, Title 40 Code of Federal Regulations, Part 265, as amended, U.S. Environmental Protection Agency, Washington, D.C.

Hartman, M. J., 1990, Results of Ground Water Quality Assessment Monitoring at the 1301-N Liquid Waste Disposal Facility and 1324-N/NA Facilities, WHC-SD-EN-EV-003, Rev. 0, Westinghouse Hanford Company, Richland, Washington.

Hartman, M. J., 1991, Groundwater Monitoring Plan for the 1301-N, 1324-N/NA, and 1325-N Facilities, WHC-SD-EN-AP-038, Rev. 0, Westinghouse Hanford Company, Richland, Washington.

Kaza, G. L., 1991, Ground Water Monitoring Plan for the 216-A-29 Ditch, WHC-SD-EN-AP-045, Rev. 0, Westinghouse Hanford Company, Richland, Washington.

Resource Conservation and Recovery Act of 1976, Public Law 94-580, 90 Stat. 2795, 42 USC 6901 et seq.

Teel, S. S., B. N. Bjornstad, and D. R. Newcomer, 1989, Interim Status GroundWater Monitoring Plan for the Grout Treatment Facility, WHC-SD-EN-AP-006, Rev. 0, Westinghouse Hanford Company, Richland, Washington.

WHC, 1991, Environmental Investigation and Site Characterization Manual, WHC-CM-7-7, Westinghouse Hanford Company, Richland, Washington. 
DOE/RL-91-47

This page intentionally left blank. 
D0E/RL-91-47

\section{DISTRIBUTION}

Number of copies

ONSITE

9

U.S. Department of EnergyRichland Operations office

R. J. Nevarez

A5-21

M. W. Tiernan

A5-55

M. J. Furman (4)

A5-21

R. A. Gilbert

A5-21

Public Reading Room (2)

A $1-65$

5

Pacific Northwest Laboratory

J. V. Borghese

K6-96

M. A. Chamness

$\mathrm{K} 6-96$

M. A. Neely

$\mathrm{K} 6-96$

R. M. Smith

K6-96

Technical Files

K1-11

1

Westinghouse Savannah River Company

R. C. Tuckfield

59

Westinghouse Hanford Company

M. R. Adams

H4-55

D. J. Brown

B4-63

J. A. Caggiano

H4-56

J. W. Cammann

C. D. Delaney

H4-14

J. J. Dorian

H4-56

D. B. Erb

K. R. Fecht

B2-16

B. H. Ford

R1-51

H4-56

E. A. Gordon

M. J. Hartman

H4-56

F. N. Hodges

$\mathrm{H} 4-17$

D. G. Horton

H4-56

R. L. Jackson

H4-56

$\mathrm{H} 4-56$

V. G. Johnson

H4-56

D. H. Jones

G. L. Kasza

$\mathrm{H} 4-56$

A. J. Knepp

H4-16

R. J. Landon

H4-56

H4-56

A. G. Law

M. Marratt

B2- 19

$\mathrm{H} 4-56$

M. M. McCarthy

H4-56

R. B. Mercer

N3-13

H4-56 
DOE/RL-91-47

\section{DISTRIBUTION (continued)}

Number of copies
M. A. Minalic
F. A. Ruck
L4-88
R. E. Peterson
H4-57
R. D. Pierce
H4-56
S. M. Price
N3-13
W. H. Price
$\mathrm{H} 4-57$
J. S. Schmid (15)
J. A. Serkowski
SO-03
$\mathrm{H} 4-56$
L. C. Swanson
H4-56
R. R. Thompson
H4 -56
D. K. Tyler
B. A. Williams
C. D. Wittreich
L4-88
H4 -56
H4-56
R. F. Wood
H4-55
J. F. Woods
R4-01
Central Files
G4-07
EDMC (3)
L8-04
Publications Services
(3)
H4-22
H4- 17 

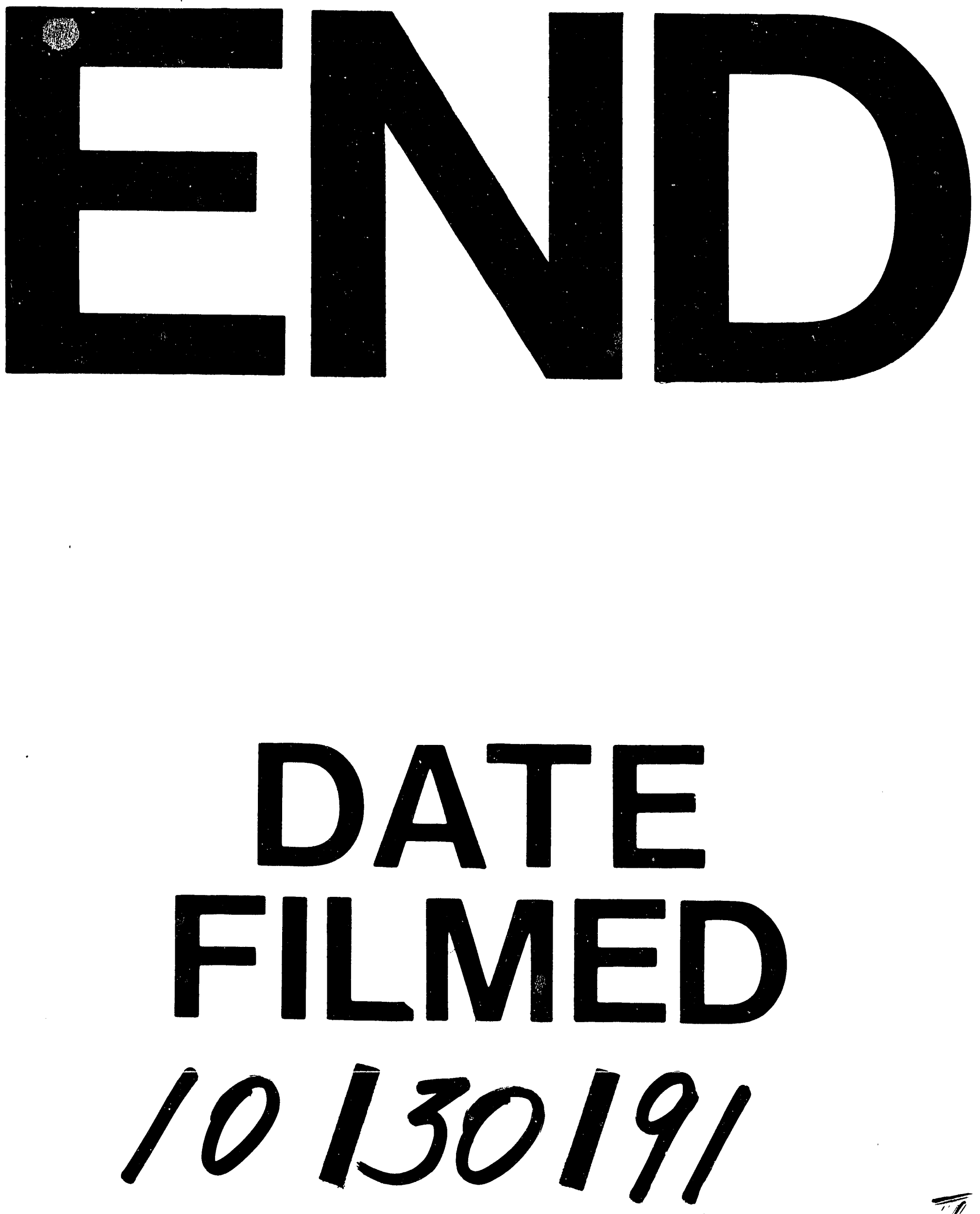
